(C) 2020 by the Arizona Board of Regents on behalf of the University of Arizona. This is an Open Access article, distributed under the terms of the Creative Commons Attribution licence (http://creativecommons. org/licenses/by/4.0/), which permits unrestricted re-use, distribution, and reproduction in any medium, provided the original work is properly cited.

\title{
NEW BAYESIAN RADIOCARBON MODELS AND CERAMIC CHRONOLOGIES FOR EARLY BRONZE IV TELL ABU EN-NI‘AJ AND MIDDLE BRONZE AGE TELL EL-HAYYAT, JORDAN
}

\author{
Patricia L Fall $^{1 *(1)} \cdot$ Steven E Falconer ${ }^{2} \cdot$ Felix Höflmayer $^{3}$ \\ ${ }^{1}$ Department of Geography \& Earth Sciences, University of North Carolina Charlotte, Charlotte, NC 28223, USA \\ ${ }^{2}$ Department of Anthropology, University of North Carolina Charlotte, Charlotte, NC 28223, USA \\ ${ }^{3}$ Austrian Academy of Sciences, Institute for Oriental and European Archaeology, Vienna, Austria
}

\begin{abstract}
We present two new Bayesian ${ }^{14} \mathrm{C}$ models using IntCal20 that incorporate 17 new calibrated AMS ages for Early Bronze IV Tell Abu en-Ni‘aj and Middle Bronze Age Tell el-Hayyat, located in the northern Jordan Valley, Jordan. These freshly augmented suites of carbonized seed dates now include 25 AMS dates from Tell Abu en-Ni'aj and 31 AMS dates from Tell el-Hayyat. The modeled founding date for Tell Abu en-Ni'aj strengthens an emerging high chronology for Early Bronze IV starting by $2500 \mathrm{cal}$ BC, while the end of its habitation by 2200 cal BC may exemplify a regional pattern of increasingly pervasive abandonment among late Early Bronze IV settlements in the Southern Levant. In turn, our modeled date for the Early Bronze IV/Middle Bronze Age transition at Tell el-Hayyat around 1900 cal BC pushes this interface about a century later than surmised traditionally, and its abandonment in Middle Bronze III marks an unexpectedly early end date before 1600 cal BC. These inferences, which coordinate Bayesian AMS models and typological ceramic sequences for Tell Abu en-Ni‘aj and Tell el-Hayyat, contribute to an ongoing revision of Early and Middle Bronze Age Levantine chronologies and uncoupling of their attendant interpretive links between the Southern Levant and Egypt.
\end{abstract}

KEYWORDS: AMS chronology, Bayesian modeling, Bronze Age, ceramic chronology, Jordan Valley, Levant.

\section{INTRODUCTION}

The Early and Middle Bronze Ages of the Southern Levant are characterized traditionally in terms of early urbanism, its abandonment and redevelopment, between about 3500 and 1500 BC. Incipient walled settlements emerged in Early Bronze I (Joffe 1993; Gophna 1995; Philip 2003), followed by larger and more numerous fortified towns in Early Bronze II-III, time periods often combined to reflect a lengthy initial era of Levantine urbanism (Greenberg 2002, 2014; Philip 2008; de Miroschedji 2009, 2014). The ensuing Early Bronze IV Period (or Intermediate Bronze Age) witnessed the pervasive abandonment of these towns in the Southern Levant. Traditional social interpretations have emphasized seasonal transhumant pastoralism (e.g., between low elevation encampments and hill country cemeteries; Dever 1980, 2014), while more recent studies have elucidated a growing number of sedentary agrarian villages (Palumbo 1991; Cohen 2009; Falconer and Fall 2009, 2019; Richard et al. 2010; Prag 2001, 2014; D’Andrea 2014). The subsequent Middle Bronze Age has long been celebrated as the apex of pre-Roman urbanism in the Southern Levant (e.g., Dever 1987; Ilan 1995). Walled cities reappeared rapidly in Middle Bronze I (known previously as Middle Bronze IIA) and grew in size, number, and fortification during Middle Bronze II and III (formerly Middle Bronze IIB and IIC) (Burke 2008; Bourke 2014; Cohen 2014). In overview, Levantine urbanization has been viewed as a long-term social transformation that unfolded over most of the third and second millennia BC, aside from a brief punctuation during the abandonment of towns in Early Bronze IV (hence this period's alternative "intermediate" nomenclature).

*Corresponding author. Email: pfall@uncc.edu. 
The relative chronology of the Southern Levantine Bronze Age traditionally derives from seriated material culture sequences and stylistic parallels (especially in pottery vessel morphology and metal weaponry) with Syria and Lebanon (Cohen 2002, 2014; Bourke 2014; de Miroschedji 2014; Prag 2014; Richard 2014). Major junctures in Levantine absolute chronology and their associated socio-political interpretations have relied on correlations with Egyptian dynastic history: (1) Early Bronze II-III town life roughly paralleled the rise of the Egyptian Old Kingdom (Stager 1992; Bruins and van der Plicht 2001; Sowada 2009); (2) Early Bronze IV town abandonment is correlated with political decentralization during the Egyptian First Intermediate Period ca. 2300/2200 to 2000 BC (Stager 1992; Dever 1995; Prag 2014); (3) the rapid reestablishment of Middle Bronze Age towns is both inferred and explained as a response to political reunification starting with the ascension of the Egyptian 12th Dynasty ca. 2000 BC (Dever 1987; Stager 1992; Cohen 2016; see critique in Bruins 2007); and (4) the apex of Middle Bronze urbanism (MB III) is correlated with Hyksos rule in Egypt, ending with the Hyksos "expulsion" ca. 1550/1500 BC (Bietak 2013; Burke 2014; Sharon 2014; see discussion in Höflmayer 2019).

As a result of an ongoing radiocarbon revolution (e.g., see Manning et al. 2014) involving sitespecific and regional Bayesian radiocarbon modeling (Bronk Ramsey 2009a), the chronology and interpretation of early Levantine urban growth and abandonment are undergoing substantial revision. Critical examination of Levantine radiocarbon chronologies now reveals that former correlations with, and interpretations based on, Egyptian dynastic history may no longer be assumed axiomatically (Kutschera et al. 2012), whereas the Egyptian historical chronology itself has proven to be compatible with radiocarbon dating (Bronk Ramsey et al. 2010). For example, earlier beginning and end dates have been proposed for Early Bronze II and III as part of an emerging "high" Early Bronze chronology (e.g., Bruins and van der Plicht 2001; Golani and Segal 2002; Bourke et al. 2009; Regev et al. 2012a, 2014; Höflmayer et al. 2014; Falconer and Fall 2016), while a shortened duration has been suggested for the Middle Bronze Age (e.g., Höflmayer et al. 2016a; Falconer and Fall 2016, 2017).

At the heart of these revisions lie three crucial temporal junctures: the earliest evidence for Early Bronze IV village settlements, the Early Bronze IV/Middle Bronze Age interface between eras of town abandonment and rejuvenation, and the end of the Middle Bronze Age II-III apex of town life. Excavations at the village sites of Tell Abu en-Ni'aj and Tell el-Hayyat, located in close proximity in the northern Jordan Valley (Figure 1), provide detailed stratified settlement histories and fine grained radiocarbon chronologies through Early Bronze IV and the Middle Bronze Age. New Bayesian models based on expanded suites of seed ages from both sites illuminate these junctures in the northern Jordan Valley, as well as their larger implications for independent explanation of Bronze Age societal dynamics in the Southern Levant.

\section{SITE DESCRIPTIONS}

\section{Tell Abu en-Ni‘aj}

Tell Abu en-Ni ${ }^{\prime}$ aj $\left(32^{\circ} 24^{\prime} 52^{\prime \prime} \mathrm{N} ; 35^{\circ} 34^{\prime} 6^{\prime \prime} \mathrm{E}\right)$ incorporates the remains of an Early Bronze IV agrarian village in the northern Jordan Valley, Jordan. This mound sits at about $250 \mathrm{~m}$ below sea level (bsl) overlooking the zor, the active floodplain of the Jordan River, from its position near the edge of the ghor, the terrace of agricultural lands to the east. This site's size of about 2.5 ha suggests a likely Bronze Age population of 500-600 people (Falconer and Fall 2019), 
(A)

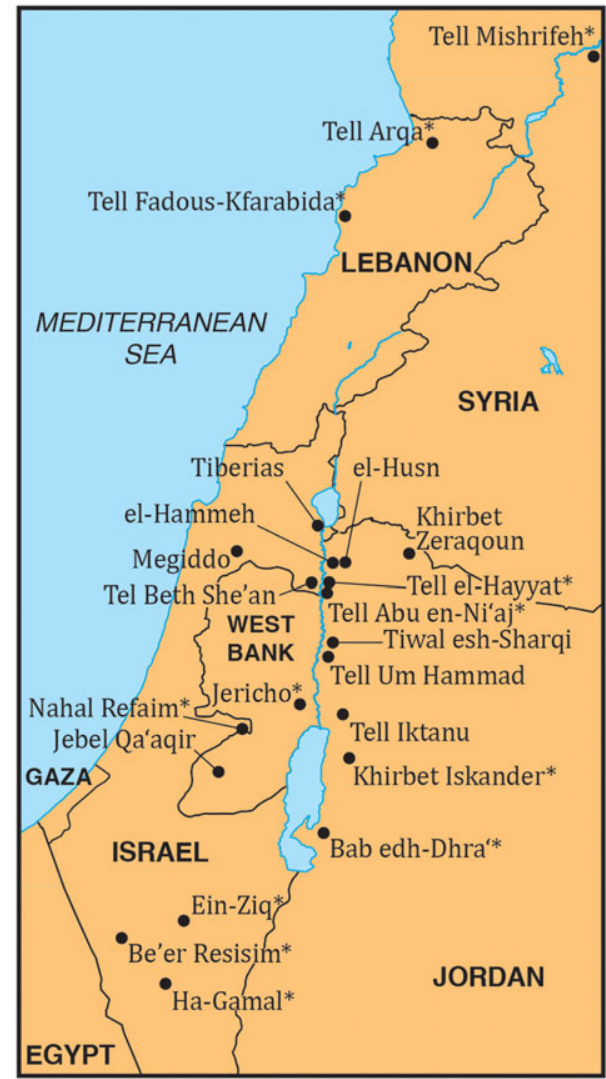

(B)
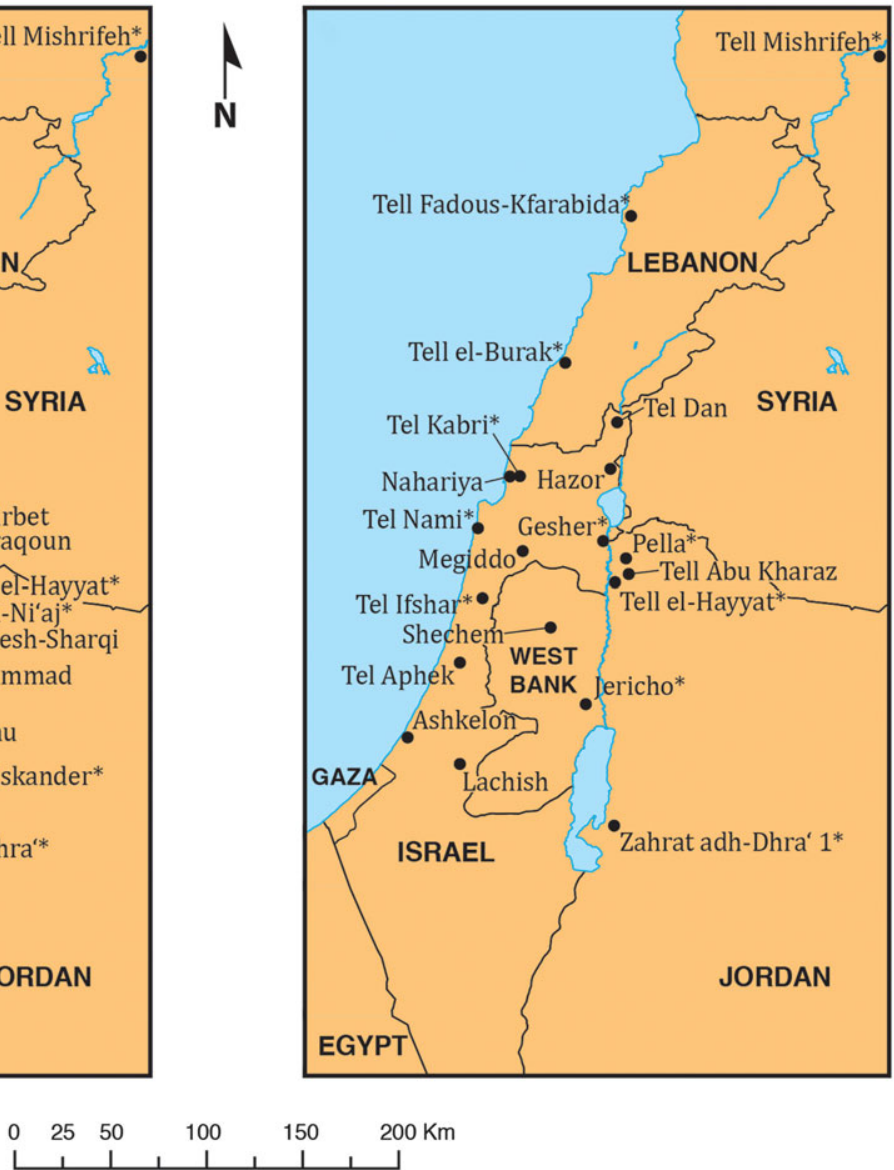

Figure 1 Maps of the Eastern Mediterranean showing (A) Early Bronze IV and (B) Middle Bronze Age archaeological sites that contribute to the regional radiocarbon and ceramic chronologies. Sites with an asterisk have contributed radiocarbon ages.

based on analogies with the population densities of ethnographically documented traditional farming villages in southwestern Asia (e.g., Kramer 1982). Tell Abu en-Ni'aj rises $3.30 \mathrm{~m}$ above the surrounding landscape, which consisted of agricultural fields during the site's excavation. Today, the tell is fenced amid the roads and warehouses of the Jordan Gateway industrial park. The site was reported originally by the East Jordan Valley Survey as being relatively large with predominantly "EB-MB" surface ceramics (Ibrahim et al. 1976: 49, 51; site 64). Tell Abu enNi'aj, occupied solely during the EB IV Period, is particularly significant as an uncommon example of a sedentary, continuously occupied, agriculturally dedicated settlement in a time period traditionally interpreted by archaeologists in terms of non-sedentary pastoral society.

\section{Tell el-Hayyat}

Tell el-Hayyat $\left(32^{\circ} 25^{\prime} 14^{\prime \prime} \mathrm{N} ; 35^{\circ} 34^{\prime} 36^{\prime \prime} \mathrm{E}\right)$ lies approximately $1.5 \mathrm{~km}$ northeast of Tell Abu en-Ni'aj at an elevation of about $240 \mathrm{~m}$ bsl in the midst of orchards and cultivated fields in the ghor. This 0.5 ha mound incorporates about $4.50 \mathrm{~m}$ of archaeological deposits and 
embodies the remains of a Bronze Age community of up to 200 inhabitants (Falconer and Fall 2006). Tell el-Hayyat was reported originally among the sites surveyed by Glueck (1951: 259; site 154, "Tell abu Hayet") and by Mellaart (1962: 144-145; site 24, "Tell Abu Hayet"). The subsequent, more systematic East Jordan Valley Survey highlighted Tell el-Hayyat's evidence for occupation during "EB-MB, MB IIA, MB IIB-C" (Ibrahim et al. 1976: 49; site 56) and therefore emphasized the site as having the potential to provide "a stratigraphically controlled sequence from EB-MB to MB IIB-C" (Ibrahim et al. 1976: 54). Indeed, a major part of Tell el-Hayyat's significance lies in the chronological inferences we can draw from its rare stratigraphic sequence over the Early Bronze IV/Middle Bronze Age transition and its record of agrarian village life during Middle Bronze I-III.

\section{METHODS}

\section{Field Excavations}

Tell Abu en-Ni'aj was excavated over three field seasons totaling 16 weeks in fall 1985, winter 1996/97 and winter/spring 2000 (Falconer and Fall 2019). Our initial field season involved two weeks of test excavations in ten $4 \times 4$ m units in Fields 1-3, primarily exposing Phases 3-2 with soundings to Phase 6 (Figure 2). Two subsequent seasons featured excavation of 27 contiguous $4 \times 4 \mathrm{~m}$ units in Field 4 through seven stratified architectural phases of occupation, from uppermost Phase 1 to basal Phase 7. Fifteen units reached archaeologically sterile sediments underlying Phase 7. The cumulative area of the excavation units in all four fields represents about $2.5 \%$ of the site's area.

Tell el-Hayyat was excavated in three field seasons totaling 24 weeks in 1982, 1983 and 1985 (Falconer and Fall 2006). Excavation of $164 \times 4 \mathrm{~m}$ units and four $2 \times 4 \mathrm{~m}$ units proceeded through six stratigraphic phases from uppermost Phase 1 to basal Phase 6 (Figure 3). Units A and $\mathrm{B}$ uncovered an intact pottery kiln and ceramic production debris on the south flank of the tell, while Units C-U revealed village remains centered around a remarkable stratified sequence of four Canaanite temples in antis (Magness-Gardiner and Falconer 1994; Falconer and Fall 2006). The aggregate excavated exposure of these 20 units represents about $8.0 \%$ of the site's area.

\section{Material Culture Recovery and Analysis}

During the excavation of both sites, larger elements of material culture and animal bones were recovered in the course of excavation, and all excavated sediments (other than those processed by water flotation) were dry-sieved in the field through $0.5 \mathrm{~cm}$ wire mesh to insure maximum recovery of smaller sized ceramics, bones, metal and stone artifacts. All excavated remains, except metallurgical evidence, were washed and sorted by material type for preliminary descriptive analysis in our field quarters at the Deir Alla Archaeological Station. All sherds were counted, non-diagnostics were returned to the modern surface of the two sites, and diagnostic sherds were labeled. Following this preliminary assessment, the excavated collections were shipped to the University of Arizona (1982-1985 seasons) and Arizona State University (1996/97 and 2000 seasons) for further analyses.

We employed the same methods of ceramic collection, analysis and interpretation at both archaeological sites (Falconer and Fall 2006: 29-30, 44-64; Falconer and Fall 2019: 13, 75-102). During the excavations, all ceramic remains were washed and sorted to segregate diagnostic sherds, including rims, bases, handles, spouts and all decorated specimens, from 


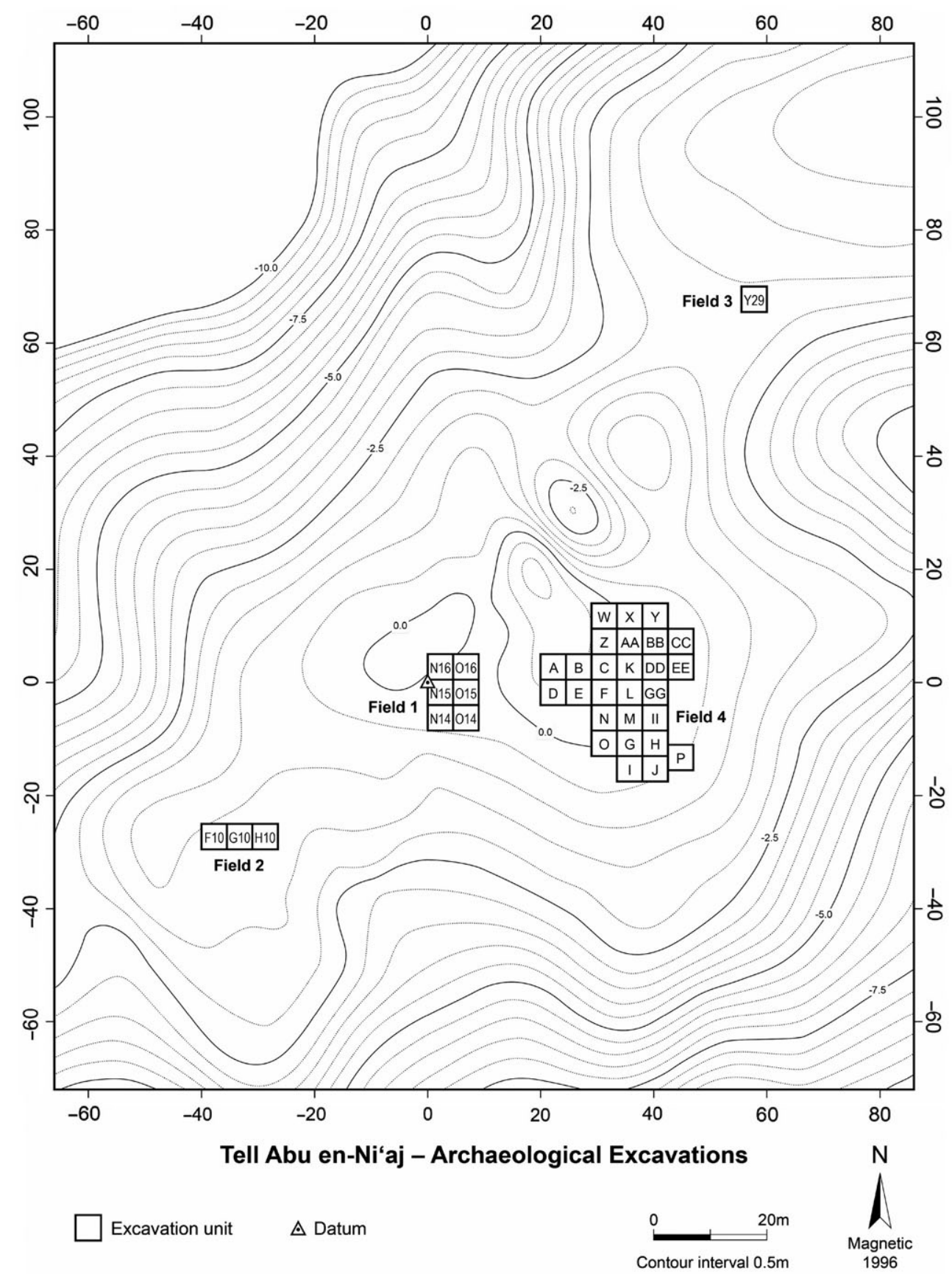

Figure 2 Excavation units at Tell Abu en-Ni‘aj in Fields 1-4; contour interval $=0.5 \mathrm{~m}$. The main datum and 0.0 contour line lie at approximately $250 \mathrm{~m}$ bsl.

non-diagnostic undecorated body sherds. During lab analysis, all diagnostic sherds were quantified according to vessel form and function, decorative techniques were coded and counted, sherd dimensions and rim and base diameters were measured. Vessel forms, and the relative frequencies of vessel sizes, morphologies and decorative techniques were 


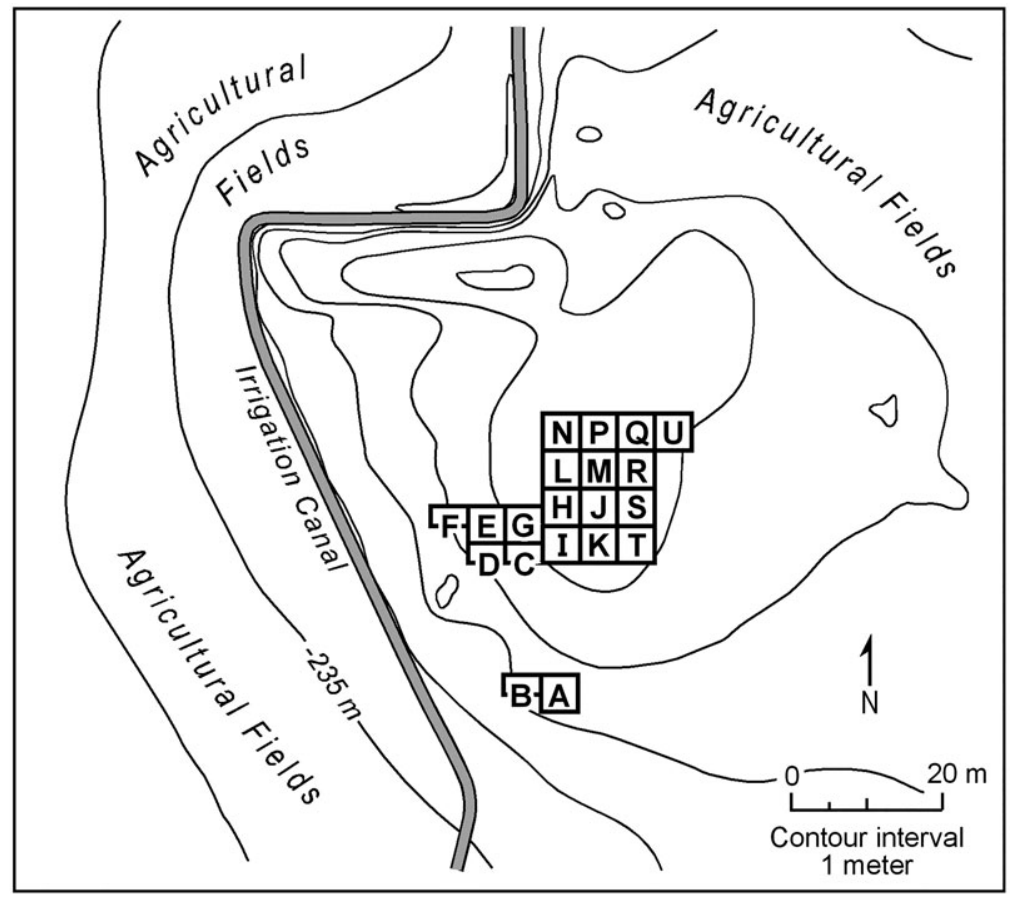

Figure 3 Excavation units at Tell el-Hayyat; contour interval $=1.0 \mathrm{~m}$.

calculated and compared through the stratigraphic sequence at each settlement and compared with evidence from assemblages at other sites to infer chronological relationships and geographical affinities with other Bronze Age settlements in the Southern Levant (see discussions in Falconer and Fall 2006: 44-64, 118-123, 2019: 75-114).

\section{Botanical Analysis}

All sediments with visible burned organic content excavated at Tell Abu en-Ni‘aj and Tell elHayyat were processed using water flotation to recover plant macrofossils (Fall et al. 1998, 2002, 2015, 2019; Falconer and Fall 2006: 38-43, 2019: 13-14; Klinge and Fall 2010). To minimize the potential for chronological mixing, samples were selected as often as possible from relatively shallow localized deposits in or on burned surfaces. During the Tell el-Hayyat excavations and the 1985 season at Tell Abu en-Ni'aj, each flotation sample was poured into a metal basket with $3.2 \mathrm{~mm}$ mesh screen across its bottom, which was suspended in a metal tub of water. Each sample was agitated to dissolve the sediment and dislodge carbonized plant fragments, which were extracted with a large tea strainer (1.6 mm mesh). The smallest seeds were recovered by placing a piece of cheese cloth in the strainer. The coarse sediment fractions at the bottom of the flotation equipment were checked after processing of each sample for occasional heavier seeds that did not float (e.g., olive stones). During the 1996/97 and 2000 seasons, a Flote-Tech 2000 flotation machine was used to separate organic remains from the sediment matrix mechanically (Figure 4). Following flotation, plant remains were dried indoors for about 24 hours before being packaged for laboratory analysis. Both the coarse and fine fractions were examined for seeds and charcoal fragments. 


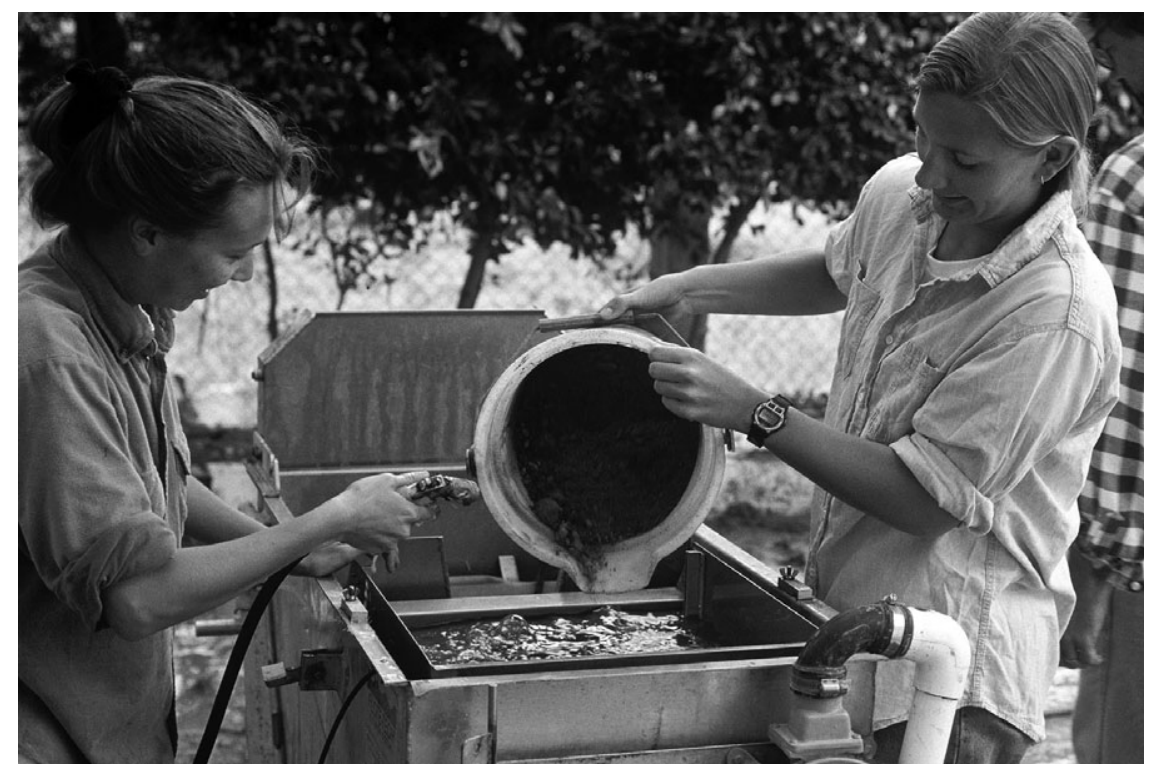

Figure 4 Recovery of plant macrofossils using Flote-Tech 2000 equipment at the Deir Alla Station during 1996/97 excavation season at Tell Abu en-Ni'aj.

In the lab, each dried sample of carbonized remains was poured through nested $4.75 \mathrm{~mm}$, $2 \mathrm{~mm}, 1 \mathrm{~mm}$ and $0.5 \mathrm{~mm}$ mesh sieves (Falconer and Fall 2006: 38-43, 2019: 13-14; Klinge and Fall 2010; Fall et al. 2015; Porson et al. 2019; Fall et al. 2019). All recovered material $0.25 \mathrm{~mm}$ or larger was sorted under a binocular microscope at 6 to $40 \times$ magnification to separate charcoal fragments from charred seeds. Seeds were identified using Fall's personal reference collection and comparative literature (e.g., Helbaek 1958, 1966; Delorit 1970; Martin and Barkley 1973; Zohary and Hopf 1973; van Zeist 1976; Zohary and Spiegel-Roy 1975; van Zeist and Bakker-Heeres 1982; Hubbard 1992; Jacomet 2006), counted and categorized taxonomically (Klinge and Fall 2010; Porson et al. 2019; Fall et al. 2019). Analysis of 123 flotation samples from Tell Abu en-Ni'aj has led to the recovery of more than 20,000 carbonized seeds, seed fragments and chaff (Porson et al. 2019), and 152 samples from Tell el-Hayyat have produced nearly 9000 identified seeds (Falconer and Fall 2006: 65-72; Fall et al. 2019).

\section{AMS Analysis}

Over the course of our investigations, we have emphasized the importance of generating AMS ages from seeds, rather than charcoal specimens, which are susceptible to the effects of inbuilt age (Waterbolk 1971). Our radiocarbon ages have been determined by the University of Arizona Accelerator Mass Spectrometry Laboratory, the University of Groningen Centre for Isotope Research, the Vienna Environmental Research Accelerator and the Oxford Radiocarbon Accelerator Unit.

Seed samples from Tell Abu en-Ni'aj were selected from well-defined contexts in all seven stratigraphic phases for AMS analysis (Table 1). Our sampling focused on cereals identified as Triticum sp. or Hordeum sp. whenever possible, and also included two samples of Prosopis sp. seeds. Among these 25 samples, 23 are drawn from small, well-defined burned 
Table 1 AMS radiocarbon results for seed samples from Tell Abu en-Ni'aj, Jordan. Calibration based on OxCal 4.4.2 (Bronk Ramsey 2009a, 2017) using the IntCal20 atmospheric curve (Reimer et al. 2020). Stratigraphic phases at Tell Abu en-Ni'aj start with Phase 7 (the earliest, basal stratum) and end with Phase 1 (the latest, uppermost stratum). Samples are tabulated by phase and ordered chronologically according to conventional ${ }^{14} \mathrm{C}$ age within each phase. Context is indicated according to Excavation Unit, Locus and Bag (e.g., D.016.51 = Unit D, Locus 016, Bag 51); * Outlier Index $\geq 5 \%$.

\begin{tabular}{|c|c|c|c|c|c|}
\hline$\#$ & Lab number & $\begin{array}{l}\text { Conventional } \\
{ }^{14} \mathrm{C} \text { age yr BP }\end{array}$ & $\begin{array}{l}\text { Calibrated } 1 \sigma \text { ranges } \\
\text { yr BC (probability) }\end{array}$ & $\begin{array}{c}\text { Calibrated } 2 \sigma \text { ranges } \\
\text { yr BC (probability) }\end{array}$ & Archaeological context \\
\hline$\overline{25}$ & AA-113005 & $3804 \pm 25$ & $2287-2201(68.3 \%)$ & $\begin{array}{c}2341-2316(3.6 \%) \\
2310-2191(78.1 \%) \\
2181-2142(13.7 \%)\end{array}$ & $\begin{array}{l}\text { Phase 1, D.016.51, clay bin } \\
\text { Hordeum seeds }\end{array}$ \\
\hline 24 & AA-113006 & $3835 \pm 32$ & $\begin{array}{l}2343-2271(36.6 \%) \\
2260-2205(31.7 \%)\end{array}$ & $\begin{array}{c}2454-2418(6.6 \%) \\
2409-2199(87.1 \%) \\
2163-2151(1.7 \%)\end{array}$ & $\begin{array}{l}\text { Phase } 1, \text { E. } 016.82 \text {, pit } \\
\text { Hordeum seeds }\end{array}$ \\
\hline 23 & AA-113003 & $3836 \pm 25$ & $\begin{array}{c}2341-2317(13.5 \%) \\
2309-2275(21.0 \%) \\
2256-2206(33.9)\end{array}$ & $\begin{array}{c}2452-2420(4.5 \%) \\
2407-2375(7.7 \%) \\
2354-2200(83.3 \%)\end{array}$ & $\begin{array}{l}\text { Phase } 1, \text { C. } 015.50 \text {, pit } \\
\text { Hordeum seeds }\end{array}$ \\
\hline 22 & AA-113004 & $3861 \pm 25$ & $\begin{array}{c}2451-2421(11.8 \%) \\
2406-2377(14.5 \%) \\
2351-2287(39.1 \%) \\
2246-2239(2.8 \%)\end{array}$ & $\begin{array}{l}2457-2281(84.7 \%) \\
2252-2230(7.1 \%) \\
2223-2209(3.6 \%)\end{array}$ & $\begin{array}{l}\text { Phase } 1 \text {, D. } 009.41 \text {, mudbrick debris } \\
\text { Hordeum seeds }\end{array}$ \\
\hline 21 & VERA-2043 & $3810 \pm 35$ & $\begin{array}{c}2296-2198(61.4 \%) \\
2166-2150(6.9 \%)\end{array}$ & $\begin{array}{c}2450-2422(2.1 \%) \\
2406-2377(3.6 \%) \\
2351-2138(89.8 \%)\end{array}$ & $\begin{array}{l}\text { Phase 2, C. } 037.126 \text {, fire pit } \\
\text { Hordeum seeds, humic acids }\end{array}$ \\
\hline 20 & VERA-2042 & $3820 \pm 35$ & $\begin{array}{c}2340-2322(7.1 \%) \\
2304-2200(61.2 \%)\end{array}$ & $\begin{array}{c}2454-2419(4.0 \%) \\
2408-2373(5.9 \%) \\
2356-2192(77.2 \%) \\
2181-2142(8.3 \%)\end{array}$ & $\begin{array}{l}\text { Phase } 2, \text { B. } 024.172 \text {, ash pit } \\
\text { Hordeum seeds }\end{array}$ \\
\hline 19 & VERA-2044 & $3830 \pm 40$ & $\begin{array}{c}2396-2388(2.4 \%) \\
2344-2202(65.9 \%)\end{array}$ & $\begin{array}{l}2456-2196(90.3 \%) \\
2172-2146(5.1 \%)\end{array}$ & $\begin{array}{l}\text { Phase } 2, \text { B. } 010.063 \text {, fire pit } \\
\text { Triticum and Hordeum seeds, } \\
\text { humic acids }\end{array}$ \\
\hline 18 & OxA-10991 & $3877 \pm 40$ & $\begin{array}{l}2454-2418(17.7 \%) \\
2409-2370(19.2 \%) \\
2361-2296(31.4 \%)\end{array}$ & $\begin{array}{l}2467-2276(85.0 \%) \\
2256-2207(10.4 \%)\end{array}$ & $\begin{array}{l}\text { Phase } 2, \text { B. } 024.172 \text {, ash pit } \\
\text { Hordeum seeds }\end{array}$ \\
\hline
\end{tabular}


Table 1 (Continued)

\begin{tabular}{|c|c|c|c|c|c|}
\hline \# & Lab number & $\begin{array}{l}\text { Conventional } \\
{ }^{14} \mathrm{C} \text { age yr BP }\end{array}$ & $\begin{array}{l}\text { Calibrated } 1 \sigma \text { ranges } \\
\text { yr BC (probability) }\end{array}$ & $\begin{array}{l}\text { Calibrated } 2 \sigma \text { ranges } \\
\text { yr BC (probability) }\end{array}$ & Archaeological context \\
\hline 17 & OxA-10992 & $3886 \pm 40$ & $\begin{array}{l}2457-2340(61.3 \%) \\
2320-2306(7.0 \%)\end{array}$ & $\begin{array}{l}2469-2280(88.1 \%) \\
2253-2209(7.4 \%)\end{array}$ & $\begin{array}{l}\text { Phase 2, B. } 010.063 \text {, fire pit } \\
\text { Cerealia seeds }\end{array}$ \\
\hline 16 & AA-94179 & $3828 \pm 39$ & 2343-2202 (68.3\%) & $\begin{array}{l}2455-2417(6.8 \%) \\
2410-2196(82.9 \%) \\
2174-2146(5.7 \%)\end{array}$ & $\begin{array}{l}\text { Phase } 3 \text {, GG. } 015.49 \text {, burned surface } \\
\text { Prosopis seeds }\end{array}$ \\
\hline 15 & VERA-2041 & $3900 \pm 50$ & $\begin{array}{l}2464-2339(60.4 \%) \\
2322-2303(7.9 \%)\end{array}$ & $\begin{array}{c}2560-2539(2.0 \%) \\
2491-2274(85.6 \%) \\
2257-2206(7.8 \%)\end{array}$ & $\begin{array}{l}\text { Phase } 3, \text { K.018.030, clay-lined pit } \\
\text { Hordeum seeds }\end{array}$ \\
\hline 14 & OxA-10990 & $3932 \pm 38$ & $\begin{array}{l}2474-2397(44.7 \%) \\
2387-2344(23.5 \%)\end{array}$ & $\begin{array}{l}2566-2531(7.8 \%) \\
2496-2295(87.6 \%)\end{array}$ & $\begin{array}{l}\text { Phase } 3, \text { K.018.030, clay-lined pit } \\
\text { Hordeum vulgare seeds }\end{array}$ \\
\hline 13 & AA-90070 & $3870 \pm 42$ & $\begin{array}{l}2454-2418(15.9 \%) \\
2409-2291(52.4 \%)\end{array}$ & $\begin{array}{l}2466-2271(81.5 \%) \\
2260-2204(14.0 \%)\end{array}$ & $\begin{array}{l}\text { Phase } 4, \text { C. } 073.284, \text { ash pit } \\
\text { Triticum dicoccum seeds }\end{array}$ \\
\hline 12 & AA-90069 & $3896 \pm 42$ & $\begin{array}{l}2462-2341(64.8 \%) \\
2317-2309(3.5 \%)\end{array}$ & $\begin{array}{l}2475-2276(89.1 \%) \\
2256-2206(6.4 \%)\end{array}$ & $\begin{array}{l}\text { Phase } 4 \text {, C. } 071.236 \text {, burned surface } \\
\text { Hordeum seeds }\end{array}$ \\
\hline 11 & AA-90067* & $3986 \pm 56$ & $\begin{array}{l}2580-2454(64.1 \%) \\
2418-2409(2.1 \%) \\
2371-2361(2.1 \%)\end{array}$ & $\begin{array}{l}2835-2819(0.9 \%) \\
2666-2646(1.3 \%) \\
2636-2297(93.3 \%)\end{array}$ & $\begin{array}{l}\text { Phase } 4, \text { C. } 066.239 \text {, mudbrick debris } \\
\text { Hordeum, Cerealia seeds }\end{array}$ \\
\hline 10 & AA-94178 & $3912 \pm 39$ & $\begin{array}{l}2467-2396(42.2 \%) \\
2388-2344(26.1 \%)\end{array}$ & $\begin{array}{l}2560-2539(2.0 \%) \\
2491-2285(92.5 \%) \\
2248-2237(1.0 \%)\end{array}$ & $\begin{array}{l}\text { Phase } 5 \text {, GG. } 065.185 \text {, ash lens } \\
\text { Hordeum seeds }\end{array}$ \\
\hline 9 & AA-94180 & $3915 \pm 39$ & $\begin{array}{l}2468-2396(42.6 \%) \\
2388-2344(25.7 \%)\end{array}$ & $\begin{array}{c}2562-2537(2.7 \%) \\
2493-2286(92.2 \%) \\
2246-2239(0.6 \%)\end{array}$ & $\begin{array}{l}\text { Phase 5, GG.100.289, fire pit } \\
\text { Prosopis seeds }\end{array}$ \\
\hline 8 & AA-90073 & $3915 \pm 44$ & 2469-2342 (68.3\%) & $\begin{array}{c}2566-2532(4.2 \%) \\
2495-2282(88.7 \%) \\
2251-2232(1.8 \%) \\
2220-2210(0.8 \%)\end{array}$ & $\begin{array}{l}\text { Phase } 5, \text { C. } 089.386 \text {, ash pit } \\
\text { Hordeum seeds }\end{array}$ \\
\hline
\end{tabular}


Table 1 (Continued)

\begin{tabular}{|c|c|c|c|c|c|}
\hline \# & Lab number & $\begin{array}{l}\text { Conventional } \\
{ }^{14} \mathrm{C} \text { age yr BP }\end{array}$ & $\begin{array}{l}\text { Calibrated } 1 \sigma \text { ranges } \\
\text { yr BC (probability) }\end{array}$ & $\begin{array}{l}\text { Calibrated } 2 \sigma \text { ranges } \\
\text { yr BC (probability) }\end{array}$ & Archaeological context \\
\hline 7 & AA-90071* & $3981 \pm 44$ & $\begin{array}{l}2571-2515(39.2 \%) \\
2502-2462(29.0 \%)\end{array}$ & $\begin{array}{c}2623-2596(2.5 \%) \\
2385-2395(86.2 \%) \\
2389-2344(6.8 \%)\end{array}$ & $\begin{array}{l}\text { Phase } 5 \text {, C. } 075.278 \text {, burned surface } \\
\text { Hordeum seeds }\end{array}$ \\
\hline 6 & AA-90076 & $3905 \pm 45$ & $\begin{array}{c}2465-2341(64.5 \%) \\
2317-2308(3.7 \%)\end{array}$ & $\begin{array}{c}2558-2541(1.6 \%) \\
2490-2280(88.7 \%) \\
2253-2209(5.1 \%)\end{array}$ & $\begin{array}{l}\text { Phase } 6, \text { C. } 106.494 \text {, stone hearth } \\
\text { Triticum dicoccum seeds }\end{array}$ \\
\hline 5 & AA-107228 & $3924 \pm 25$ & $\begin{array}{l}2469-2435(26.9 \%) \\
2426-2403(17.6 \%) \\
2380-2349(23.8 \%)\end{array}$ & $\begin{array}{l}2475-2336(90.2 \%) \\
2328-2298(5.3 \%)\end{array}$ & $\begin{array}{l}\text { Phase 6, C. } 111.548 \text {, pit } \\
\text { Hordeum, Triticum, Pisum seeds }\end{array}$ \\
\hline 4 & AA-107227 & $3937 \pm 24$ & $\begin{array}{l}2473-2439(31.9 \%) \\
2424-2404(15.1 \%) \\
2379-2350(21.2 \%)\end{array}$ & $\begin{array}{c}2563-2536(5.5 \%) \\
2493-2342(89.2 \%) \\
2316-2310(0.8 \%)\end{array}$ & $\begin{array}{l}\text { Phase 6, GG.098.295, pit } \\
\text { Hordeum, Triticum, Pisum seeds }\end{array}$ \\
\hline 3 & AA-90072 & $3986 \pm 44$ & $\begin{array}{l}2571-2516(40.4 \%) \\
2501-2464(27.9 \%)\end{array}$ & $\begin{array}{l}2624-2399(90.4 \%) \\
2384-2346(5.0 \%)\end{array}$ & $\begin{array}{l}\text { Phase } 6, \text { C. } 091.406 \text {, burned surface } \\
\text { Triticum dicoccum seeds }\end{array}$ \\
\hline 2 & AA-90075* & $4026 \pm 43$ & $2580-2472(68.3 \%)$ & $\begin{array}{l}2843-2814(2.9 \%) \\
2673-2461(92.6 \%)\end{array}$ & $\begin{array}{l}\text { Phase } 6, \text { C. } 086.387 \text {, burned surface } \\
\text { Cerealia seeds }\end{array}$ \\
\hline 1 & AA-94177 & $4046 \pm 39$ & $\begin{array}{l}2626-2556(35.8 \%) \\
2544-2488(32.5 \%)\end{array}$ & $\begin{array}{c}2846-2812(5.4 \%) \\
2742-2731(1.0 \%) \\
2675-2468(89.1 \%)\end{array}$ & $\begin{array}{l}\text { Phase } 7, \text { GG. } 105.331 \text {, fire pit } \\
\text { Cerealia seeds }\end{array}$ \\
\hline
\end{tabular}


features, including hearths, bins, shallow pits, and thin sediments (e.g., ash lenses) on earthen use surfaces. The remaining two samples come from localized deposits of decomposed mudbrick sediment. Our earlier publication of radiocarbon ages from Tell Abu en-Ni‘aj (Falconer and Fall 2016) included three olive pit samples incorrectly attributed to Phase 1 at this site, which have been replaced with four new Hordeum sp. seed samples (AA-113003-113006) from secure Phase 1 contexts at Tell Abu en-Ni'aj. Phases 6-1 are now represented by three to five samples each, while the more limited Phase 7 floral assemblage provides a single AMS sample.

Similarly, 31 AMS seed samples were drawn from all six stratigraphic phases at Tell el-Hayyat (Table 2). Most AMS samples consist of Triticum sp., Hordeum sp., Cerealia and Olea sp. seeds. As at Tell Abu en-Ni'aj, these specimens were recovered from localized burned features, including ovens (tabuns), ash lenses, burned surfaces, and thin occupational sediments deposited on earthen floors. Phases 5-2 provide six to seven samples each, while Phase 6 contributes three specimens and Phase 1 offers a single sample. Our previous discussion of radiocarbon chronology at Tell el-Hayyat (Falconer and Fall 2017) concentrated on ages from Phases 5 and 4, and included two dates from Phase 6, and single dates from Phases 3, 2, and 1. This study contributes a greatly strengthened set of ${ }^{14} \mathrm{C}$ data, including 13 new ages from Phases 6, 3, and 2 produced by the Micadas AMS at Groningen (prefixed GrM in Table 2).

\section{Bayesian Analysis}

The radiocarbon ages from Tell Abu en-Ni'aj and Tell el-Hayyat were calibrated using OxCal 4.4.2 (Bronk Ramsey 2009a) and the IntCal20 atmospheric curve (Reimer et al. 2020; van der Plicht et al. 2020). The analytical tools in OxCal 4.4.2 were used for Bayesian modeling of the calibrated dates. As evidenced by its increasingly common use in archaeology, Bayesian analysis permits probabilistic modeling of large suites of calibrated ${ }^{14} \mathrm{C}$ determinations from multiple strata at a site or from multiple sites across a region. This method can incorporate prior stratigraphic information and accommodate the non-normally distributed probabilities of calibrated ${ }^{14} \mathrm{C}$ ages (Bronk Ramsey 2009a). The dates from Tell Abu en-Ni'aj and Tell el-Hayyat were organized for modeling in contiguous stratigraphic phases based on their records of continuous deposition. In each phase, the sequence of the individual samples was taken to be unknown (i.e., an unordered group of events). Outliers were detected using the Outlier Analysis tool in OxCal (Bronk Ramsey 2009b). Samples that are too old (e.g., due to residual material) or too young, and thus do not fit the prior stratigraphic information for the model are often excluded by hand, a process that can be highly subjective. In order to avoid subjective bias, we employed the Outlier Analysis in OxCal, using the General model for all short-lived samples. Outlier Analysis detects determinations that do not fit prior stratigraphic information and weighs their impact on the model accordingly. Thus, outlying dates have very little impact on the overall results (the posterior probabilities). The Outlier Index (O:xx/5) shows the expected (prior) chance of a determination being an outlier.

\section{RESULTS}

\section{Stratigraphy and Architecture}

\section{Tell Abu en-Ni'aj}

The mound of Tell Abu en-Ni'aj incorporates seven stratified phases of construction and remodeling of mudbrick and rammed earth architecture (Falconer and Fall 2019). Our excavations revealed no stone-founded architecture, but did uncover flagstone surfaces and 
Table 2 AMS radiocarbon results for seed samples from Tell el-Hayyat, Jordan. Calibration based on OxCal 4.4.2 (Bronk Ramsey 2009a, 2017) using the IntCal20 atmospheric curve (Reimer et al. 2020). Stratigraphic phases at Tell el-Hayyat start with Phase 6 (earliest, basal stratum) and end with Phase 1 (latest, uppermost stratum). Samples are tabulated by phase and ordered chronologically according to conventional ${ }^{14} \mathrm{C}$ age within each phase. Context is indicated according to Excavation Unit, Locus and Bag (e.g., L.006.9 = Unit L, Locus 006, Bag 9); * Outlier Index $\geq 5 \%$.

\begin{tabular}{|c|c|c|c|c|c|}
\hline \# & Lab number & $\begin{array}{l}\text { Conventional } \\
{ }^{14} \mathrm{C} \text { age yr BP }\end{array}$ & $\begin{array}{l}\text { Calibrated } 1 \sigma \text { ranges } \\
\text { yr BC (probability) }\end{array}$ & $\begin{array}{l}\text { Calibrated } 2 \sigma \text { ranges } \\
\text { yr BC (probability) }\end{array}$ & Archaeological context \\
\hline 31 & AA-108793 & $3352 \pm 27$ & $\begin{array}{c}1686-1609(54.0 \%) \\
1577-1561(9.7 \%) \\
1554-1546(4.5 \%)\end{array}$ & $\begin{array}{c}1736-1717(6.4 \%) \\
1692-1538(89.0 \%)\end{array}$ & $\begin{array}{l}\text { Phase 1, L.006.9, ash lens } \\
\text { Hordeum seeds }\end{array}$ \\
\hline 30 & GrM-11953* & $2451 \pm 14$ & $\begin{array}{c}740-693(30.9 \%) \\
664-647(11.3 \%) \\
549-513(19.9 \%) \\
501-486(6.2 \%)\end{array}$ & $\begin{array}{c}750-686(34.7 \%) \\
666-639(13.4 \%) \\
588-580(0.7 \%) \\
571-455(41.5 \%) \\
445-417(5.2 \%)\end{array}$ & $\begin{array}{l}\text { Phase } 2 \text {, T. } 022.55 \text {, occupational debris } \\
\text { Hordeum seeds }\end{array}$ \\
\hline 29 & GrM-12047 & $3411 \pm 13$ & $\begin{array}{c}1743-1707(43.5 \%) \\
1701-1686(20.4 \%) \\
1650-1645(4.3 \%)\end{array}$ & $1748-1631(95.4 \%)$ & $\begin{array}{l}\text { Phase } 2 \text {, I.029.89, ash lens } \\
\text { Hordeum seeds }\end{array}$ \\
\hline 28 & GrM-12033 & $3412 \pm 12$ & $\begin{array}{l}1743-1707(46.9 \%) \\
1701-1687(21.4 \%)\end{array}$ & $\begin{array}{l}1749-1665(82.9 \%) \\
1660-1631(12.5 \%)\end{array}$ & $\begin{array}{l}\text { Phase 2, S.024.51, occupational debris } \\
\text { Hordeum seeds }\end{array}$ \\
\hline 27 & GrM-12336 & $3430 \pm 18$ & $\begin{array}{c}1861-1859(1.5 \%) \\
1765-1760(3.0 \%) \\
1751-1730(21.3 \%) \\
1723-1689(42.5 \%)\end{array}$ & $\begin{array}{c}1872-1846(10.2 \%) \\
1812-1809(0.3 \%) \\
1775-1670(81.7 \%) \\
1655-1638(3.3 \%)\end{array}$ & $\begin{array}{l}\text { Phase 2, I.029.89, occupational debris } \\
\text { Hordeum seeds }\end{array}$ \\
\hline 26 & GrM-12032 & $3449 \pm 12$ & $\begin{array}{l}1866-1852(15.9 \%) \\
1769-1740(36.0 \%) \\
1711-1698(16.4 \%)\end{array}$ & $\begin{array}{c}1874-1844(23.9 \%) \\
1818-1800(4.9 \%) \\
1776-1734(43.0 \%) \\
1720-1692(23.7 \%)\end{array}$ & $\begin{array}{l}\text { Phase 2, G.043.142, tabun fill } \\
\text { Cerealia seeds }\end{array}$ \\
\hline 25 & GrM-11952 & $3461 \pm 15$ & $\begin{array}{c}1872-1847(26.0 \%) \\
1813-1807(4.1 \%) \\
1774-1742(35.5 \%) \\
1707-1703(2.8 \%)\end{array}$ & $\begin{array}{c}1878-1841(30.6 \%) \\
1825-1793(15.1 \%) \\
1781-1737(40.0 \%) \\
1716-1695(9.8 \%)\end{array}$ & $\begin{array}{l}\text { Phase 2, G.045.152, ash lens } \\
\text { Hordeum seeds }\end{array}$ \\
\hline
\end{tabular}


Table 2 (Continued)

\begin{tabular}{|c|c|c|c|c|c|}
\hline \# & Lab number & $\begin{array}{l}\text { Conventional } \\
{ }^{14} \mathrm{C} \text { age yr BP }\end{array}$ & $\begin{array}{l}\text { Calibrated } 1 \sigma \text { ranges } \\
\text { yr BC (probability) }\end{array}$ & $\begin{array}{l}\text { Calibrated } 2 \sigma \text { ranges } \\
\text { yr BC (probability) }\end{array}$ & Archaeological context \\
\hline 24 & GrM-11950 & $3483 \pm 15$ & $\begin{array}{c}1876-1862(13.0 \%) \\
1856-1843(11.4 \%) \\
1822-1795(25.6 \%) \\
1779-1766(12.3 \%) \\
1759-1751(6.0 \%)\end{array}$ & $1881-1744(95.4 \%)$ & $\begin{array}{l}\text { Phase 2, G.025.60-64, ash lens } \\
\text { Hordeum seeds }\end{array}$ \\
\hline 23 & AA-108789 & $3493 \pm 30$ & $\begin{array}{c}1881-1862(11.9 \%) \\
1856-1836(12.0 \%) \\
1830-1767(42.3 \%) \\
1758-1754(2.1 \%)\end{array}$ & $\begin{array}{c}1895-1740(93.2 \%) \\
1712-1698(2.2 \%)\end{array}$ & $\begin{array}{l}\text { Phase 2, A. } 026.13 \text {, ash lens } \\
\text { Hordeum seeds }\end{array}$ \\
\hline 22 & AA-108790 & $3475 \pm 28$ & $\begin{array}{l}1876-1843(24.5 \%) \\
1822-1796(18.7 \%) \\
1779-1746(25.1 \%)\end{array}$ & $\begin{array}{l}1885-1738(89.4 \%) \\
1715-1695(6.0 \%)\end{array}$ & $\begin{array}{l}\text { Phase } 3, \text { C. } 022.9 \text {, tabun fill } \\
\text { Hordeum seeds }\end{array}$ \\
\hline 21 & GrM-12035 & $3513 \pm 13$ & $\begin{array}{l}1885-1874(11.4 \%) \\
1845-1818(29.0 \%) \\
1801-1776(27.9 \%)\end{array}$ & $\begin{array}{l}1896-1862(19.7 \%) \\
1856-1766(75.2 \%) \\
1756-1751(0.6 \%)\end{array}$ & $\begin{array}{l}\text { Phase } 3 \text {, S. } 047.123 \text {, fill debris } \\
\text { Hordeum seeds }\end{array}$ \\
\hline 20 & GrM-12037 & $3513 \pm 13$ & $\begin{array}{l}1885-1874(11.4 \%) \\
1845-1818(29.0 \%) \\
1801-1776(27.9 \%)\end{array}$ & $\begin{array}{c}1896-1862(19.7 \%) \\
1856-1766(75.2 \%) \\
1756-1751(0.6 \%)\end{array}$ & $\begin{array}{l}\text { Phase } 3 \text {, T. } 030.100 \text {, surface } \\
\text { Hordeum seeds }\end{array}$ \\
\hline 19 & GrM-12039 & $3516 \pm 13$ & $\begin{array}{l}1887-1874(13.4 \%) \\
1845-1819(28.6 \%) \\
1800-1777(26.3 \%)\end{array}$ & $\begin{array}{l}1900-1862(21.3 \%) \\
1855-1767(74.2 \%)\end{array}$ & $\begin{array}{l}\text { Phase } 3 \text {, T. } 033.111 \text {, posthole } \\
\text { Hordeum seeds }\end{array}$ \\
\hline 18 & GrM-12034 & $3528 \pm 13$ & $\begin{array}{l}1893-1876(21.8 \%) \\
1843-1822(27.5 \%) \\
1796-1779(18.9 \%)\end{array}$ & $\begin{array}{l}1924-1871(34.6 \%) \\
1849-1773(60.9 \%)\end{array}$ & $\begin{array}{l}\text { Phase } 3, \text { D. } 033.8 \text {, surface } \\
\text { Hordeum seeds }\end{array}$ \\
\hline 17 & GrM-12356 & $3536 \pm 18$ & $\begin{array}{c}1920-1909(7.4 \%) \\
1903-1877(28.3 \%) \\
1843-1823(20.6 \%) \\
1794-1780(12.0 \%)\end{array}$ & $\begin{array}{l}1937-1871(48.7 \%) \\
1849-1773(46.7 \%)\end{array}$ & $\begin{array}{l}\text { Phase } 3 \text {, I. } 050.200 \text {, occupational debris } \\
\text { Hordeum seeds }\end{array}$ \\
\hline
\end{tabular}


Table 2 (Continued)

\begin{tabular}{|c|c|c|c|c|c|}
\hline \# & Lab number & $\begin{array}{l}\text { Conventional } \\
{ }^{14} \mathrm{C} \text { age yr BP }\end{array}$ & $\begin{array}{l}\text { Calibrated } 1 \sigma \text { ranges } \\
\text { yr BC (probability) }\end{array}$ & $\begin{array}{l}\text { Calibrated } 2 \sigma \text { ranges } \\
\text { yr BC (probability) }\end{array}$ & Archaeological context \\
\hline 16 & AA-1237* & $3280 \pm 100$ & $\begin{array}{c}1681-1654(6.1 \%) \\
1642-1442(62.1 \%)\end{array}$ & $\begin{array}{c}1875-1843(1.9 \%) \\
1821-1797(1.1 \%) \\
1778-1379(90.4 \%) \\
1345-1306(2.1 \%)\end{array}$ & $\begin{array}{l}\text { Phase } 4, \text { F. } 040.235, \text { surface } \\
\text { Olea europaea seeds }\end{array}$ \\
\hline 15 & VERA-2039 & $3495 \pm 35$ & $\begin{array}{l}1882-1863(11.9 \%) \\
1855-1767(56.4 \%)\end{array}$ & $\begin{array}{c}1921-1738(92.4 \%) \\
1714-1697(3.1 \%)\end{array}$ & $\begin{array}{l}\text { Phase } 4, \text { E. } 092 \text {, tabun fill } \\
\text { Olea europaea seed, humic acids }\end{array}$ \\
\hline 14 & VERA-2040 & $3500 \pm 35$ & $\begin{array}{l}1884-1865(12.2 \%) \\
1853-1768(56.1 \%)\end{array}$ & $\begin{array}{c}1924-1741(93.6 \%) \\
1710-1699(1.8 \%)\end{array}$ & $\begin{array}{l}\text { Phase } 4, \mathrm{~J} .074, \text { ash lens } \\
\text { Olea europaea seeds }\end{array}$ \\
\hline 13 & OxA-10988 & $3502 \pm 37$ & $\begin{array}{l}1886-1863(13.4 \%) \\
1855-1767(54.9 \%)\end{array}$ & $\begin{array}{c}1929-1740(93.4 \%) \\
1711-1699(2.1 \%)\end{array}$ & $\begin{array}{l}\text { Phase } 4, \text { E. } 092, \text { tabun fill } \\
\text { Olea europaea } \text { seed }\end{array}$ \\
\hline 12 & OxA-10989 & $3523 \pm 39$ & $\begin{array}{c}1920-1910(4.0 \%) \\
1902-1869(18.4 \%) \\
1850-1772(45.9 \%)\end{array}$ & $\begin{array}{c}1956-1742(94.9 \%) \\
1708-1701(0.6 \%)\end{array}$ & $\begin{array}{l}\text { Phase } 4, \mathrm{~J} .074, \text { ash lens } \\
\text { Olea europaea seed }\end{array}$ \\
\hline 11 & AA-1238 & $3600 \pm 60$ & $\begin{array}{c}2112-2102(2.5 \%) \\
2036-1882(65.8 \%)\end{array}$ & $\begin{array}{l}2138-1868(84.2 \%) \\
1850-1771(11.2 \%)\end{array}$ & $\begin{array}{l}\text { Phase } 4, \text { C. } 070.001 \text {, surface } \\
\text { Lens culinaris seeds }\end{array}$ \\
\hline 10 & AA-1239* & $2930 \pm 80$ & $\begin{array}{c}1258-1245(3.0 \%) \\
1230-1012(65.3 \%)\end{array}$ & $\begin{array}{l}1386-1339(4.7 \%) \\
1317-921(90.7 \%)\end{array}$ & $\begin{array}{l}\text { Phase } 5, \text { F. } 045.258 \text {, ash lens } \\
\text { Punica granatum seeds }\end{array}$ \\
\hline 9 & AA-1236 & $3460 \pm 100$ & $1891-1628(68.3 \%)$ & 2028-1519 (95.4\%) & $\begin{array}{l}\text { Phase 5, F.049.288, surface } \\
\text { Lens culinaris seeds }\end{array}$ \\
\hline 8 & OxA-10986 & $3470 \pm 36$ & $\begin{array}{c}1877-1842(22.4 \%) \\
1824-1793(17.9 \%) \\
1781-1742(24.4 \%) \\
1708-1701(3.6 \%)\end{array}$ & 1892-1687 (95.4\%) & $\begin{array}{l}\text { Phase } 5 \text {, E. } 102 \text {, ash lens } \\
\text { Triticum aestivum seeds }\end{array}$ \\
\hline 7 & OxA-10987 & $3497 \pm 37$ & $\begin{array}{l}1883-1863(12.4 \%) \\
1855-1767(55.9 \%)\end{array}$ & $\begin{array}{c}1926-1738(92.3 \%) \\
1714-1696(3.2 \%)\end{array}$ & $\begin{array}{l}\text { Phase } 5, \text { H.067, ash lens } \\
\text { Triticum aestivum seeds }\end{array}$ \\
\hline 6 & VERA-2038 & $3530 \pm 60$ & $\begin{array}{l}1945-1863(32.0 \%) \\
1855-1767(36.2 \%)\end{array}$ & $\begin{array}{c}2030-1733(92.1 \%) \\
1720-1691(3.4 \%)\end{array}$ & $\begin{array}{l}\text { Phase } 5, \text { H.067, ash lens } \\
\text { Triticum aestivum seeds }\end{array}$ \\
\hline
\end{tabular}
2036-1882 (65.8\%) 1258-1245 (3.0\%) $1230-1012(65.3 \%)$ 1317-921 (90.7\%) Lens culinaris seeds

Phase 5, E.102, ash lens 1824-1793 (17.9\%) 1781-1742 (24.4\%) (3.68-1701 $(3.6 \%)$ 1883-1863 (12.4\%) $1855-1767(55.9 \%)$ $1855-1767(36.2 \%)$

$1714-1696(3.2 \%)$

Phase 5, H.067, ash lens 
Table 2 (Continued)

\begin{tabular}{|c|c|c|c|c|c|}
\hline \# & Lab number & $\begin{array}{l}\text { Conventional } \\
{ }^{14} \mathrm{C} \text { age yr BP }\end{array}$ & $\begin{array}{l}\text { Calibrated } 1 \sigma \text { ranges } \\
\text { yr BC (probability) }\end{array}$ & $\begin{array}{l}\text { Calibrated } 2 \sigma \text { ranges } \\
\text { yr BC (probability) }\end{array}$ & Archaeological context \\
\hline 5 & VERA-2037 & $3555 \pm 40$ & $\begin{array}{c}1954-1876(50.4 \%) \\
1843-1822(10.5 \%) \\
1796-1779(7.4 \%)\end{array}$ & $\begin{array}{c}2025-1992(6.4 \%) \\
1984-1862(59.7 \%) \\
1856-1766(28.9 \%) \\
1757-1752(0.5 \%)\end{array}$ & $\begin{array}{l}\text { Phase } 5 \text {, E.102, ash lens } \\
\text { Triticum aestivum seeds, humic acids }\end{array}$ \\
\hline 4 & VERA-2038W & $3565 \pm 30$ & $\begin{array}{c}1954-1881(65.2 \%) \\
1837-1830(3.1 \%)\end{array}$ & $\begin{array}{c}2021-1995(6.4 \%) \\
1981-1873(74.6 \%) \\
1846-1817(8.8 \%) \\
1802-1776(5.7 \%)\end{array}$ & $\begin{array}{l}\text { Phase } 5, \text { H. } 067 \text {, ash lens } \\
\text { Triticum aestivum seeds }\end{array}$ \\
\hline 3 & GrM-12040 & $3524 \pm 13$ & $\begin{array}{l}1890-1875(18.4 \%) \\
1843-1821(28.6 \%) \\
1797-1779(21.3 \%)\end{array}$ & $\begin{array}{l}1921-1869(29.2 \%) \\
1850-1772(66.2 \%)\end{array}$ & $\begin{array}{l}\text { Phase } 6 \text {, J.071.256, fill debris } \\
\text { Hordeum seeds }\end{array}$ \\
\hline 2 & AA-108792 & $3588 \pm 28$ & $\begin{array}{l}2008-2004(2.8 \%) \\
1971-1895(65.5 \%)\end{array}$ & $\begin{array}{l}2028-1882(94.7 \%) \\
1836-1831(0.8 \%)\end{array}$ & $\begin{array}{l}\text { Phase } 6 \text {, H. } 073.482 \text {, surface } \\
\text { Hordeum seeds }\end{array}$ \\
\hline 1 & AA-108791 & $3593 \pm 34$ & $\begin{array}{c}2014-2000(9.2 \%) \\
1976-1897(59.0 \%)\end{array}$ & $\begin{array}{c}2113-2101(0.9 \%) \\
2036-1878(91.8 \%) \\
1841-1824(2.1 \%) \\
1790-1783(0.6 \%)\end{array}$ & $\begin{array}{l}\text { Phase } 6, \text { H. } 073.482 \text {, surface } \\
\text { Hordeum seeds }\end{array}$ \\
\hline
\end{tabular}


networks of sherd-paved streets. The initial settlement, exemplified by the remains of Phase 7 in Field 4, was founded on archaeologically sterile sediments. The uppermost village is represented by Phase 1 remains, which are found over a limited area just below the modern surface at the top of the mound. The relatively modest extent of Phase 1 evidence may reflect erosion of this uppermost layer over the last four millennia. The archaeological stratification of Tell Abu en-Ni'aj shows no evidence of a stratigraphic gap or settlement hiatus from Phase 7 to Phase 1. The village's characteristically agglutinative architecture featured localized remodeling throughout its settlement history, with more substantial episodes of structural rebuilding and street reconfiguration between phases.

The architecture of Phases 7 and 6 in Field 4 includes rammed earth and mudbrick wall foundations, which are relatively sparse in comparison with those of subsequent phases. Some Phase 7 structures have adjoining walls (Falconer and Fall 2019: figure 4.4), while the separate structures in Phase 6 include an apparent broadroom temple (see Falconer and Fall 2019: 139-142, figure 10.1). In contrast, the architecture for Phases 5-1 at Tell Abu en-Ni'aj is characterized by closely packed structures with shared walls, and included an installation of three linked mudbrick basins descending down the eastern slope of the village in Phase 3, possibly for settling potting clay (e.g., Fowler et al. 2011) or separation of olive oil (e.g., Kapellakis et al. 2008) (Figure 5). The buildings in Fields 1 and 4 are separated by repeated patterns of earthen and sherd-paved streets. Some enclosed spaces have stone pavements, and exterior features include numerous pits and postholes. Both interior and exterior settings incorporate features such as clay-lined bins and in situ ceramic vessels.

\section{Tell el-Hayyat}

The history of occupation at Tell el-Hayyat is documented in six stratified phases of archaeological deposits (Falconer and Fall 2006). Phase 6 is a basal stratum lying just above archaeologically sterile sediments at the center of the site. The sediments that constitute this phase are primarily earthen use surfaces containing ceramics that feature forms, fabrics and decoration that are exclusively Early Bronze IV and include no Middle Bronze Age sherds. Other Phase 6 evidence includes substantial chipped stone remains, and modest amounts of animal bones and carbonized seeds, with no preserved architectural features. In light of the repeated comprehensive leveling of Tell el-Hayyat for phase-byphase reconstruction in subsequent phases (see discussion below), the multiple use surfaces in this basal phase, and its substantial ceramic assemblage (nearly 1000 sherds), Phase 6 represents a clearly distinguished Early Bronze IV stratum that may also have been levelled prior to building the Middle Bronze Age structures in Phase 5. The central buildings of Tell el-Hayyat in subsequent phases are four Middle Bronze Age mudbrick temples in antis, with associated standing stones and enclosure walls, which are stratified above Phase 6 at the mound's center (Magness-Gardiner and Falconer 1994; Falconer and Fall 2006: 33-43, 83-110). This remarkable temple sequence begins with the rammed earth foundations of a modest shrine in Phase 5, which are directly overlain by the remains of successively larger and more elaborate mudbrick, plastered and painted temples in Phases 4-2 (Figure 6). Although no additional Phase 5 structures were uncovered, Phase 4-2 domestic buildings with walled courtyards, which were separated by alleyways, lay outside the temple enclosures. Noteworthy architectural evidence also includes an intact pottery kiln with an interred human skull in Phase 4 (Area A; Falconer and Fall 2006: figures 3.12-3.17) and a completely burned chaff-plastered room interior in Phase 3 (Falconer and Fall 2006: East Building in figures 2.21 and 3.18). Phase 1 deposition, again largely at the center of the mound, includes fragmentary stone wall foundations 


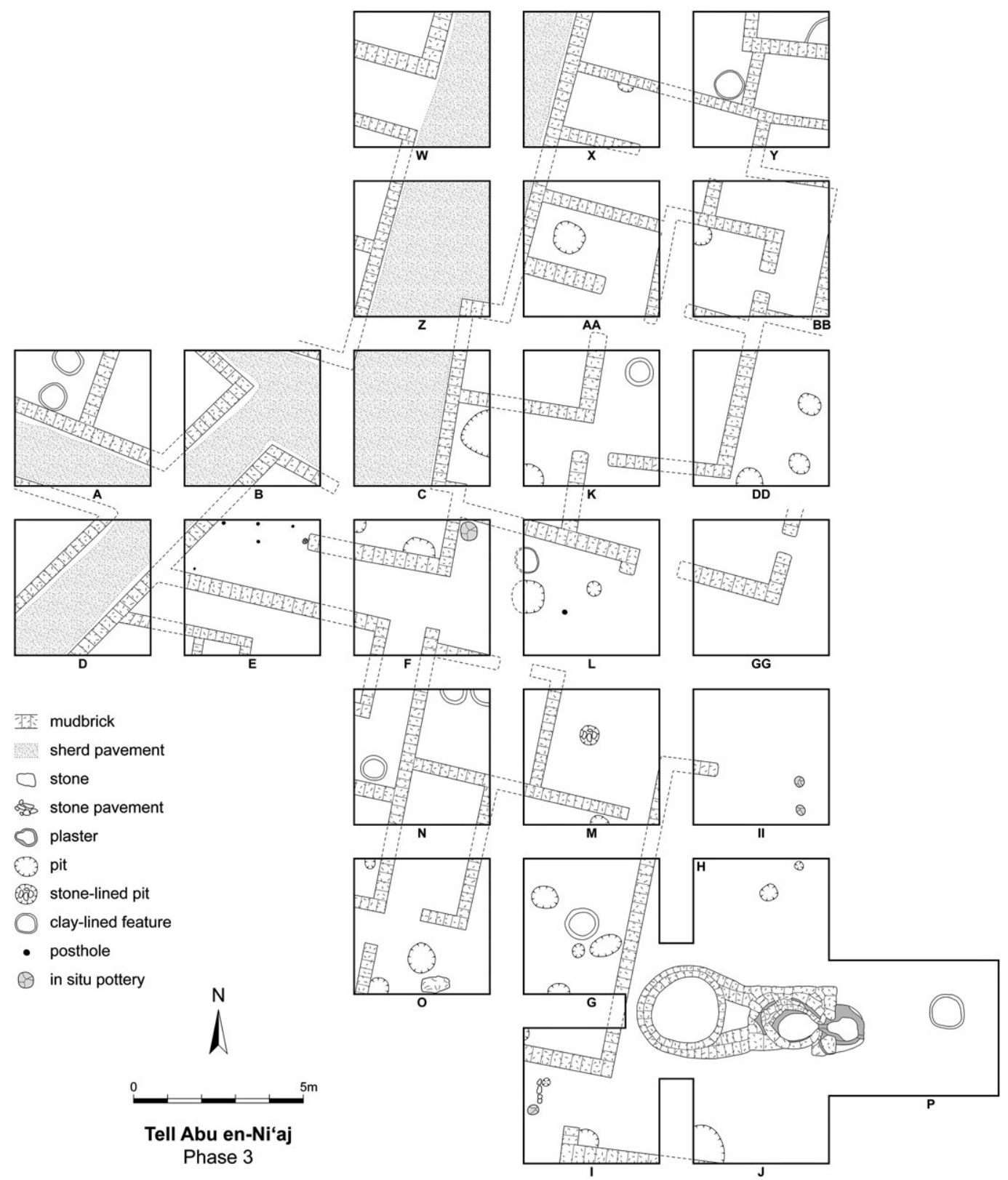

Figure 5 Phase 3 architecture in Field 4 at Tell Abu en-Ni‘aj, showing mudbrick structures, sherd-paved streets and features, including a three-chambered settling basin in Units G-J.

(but no evidence of a temple), associated use surfaces and limited amounts of pottery, chipped stone, animal bones and carbonized plant remains. The sediments of Phases 2 and 1 are punctuated by several post-Bronze Age pits (Falconer and Fall 2006: 60, figure 4.15: a, b). The construction material in all phases at Tell el-Hayyat is mudbrick, aside from stone foundations for the temples in Phases 3 and 2, and for the domestic structures in Phases 2 and 1. 


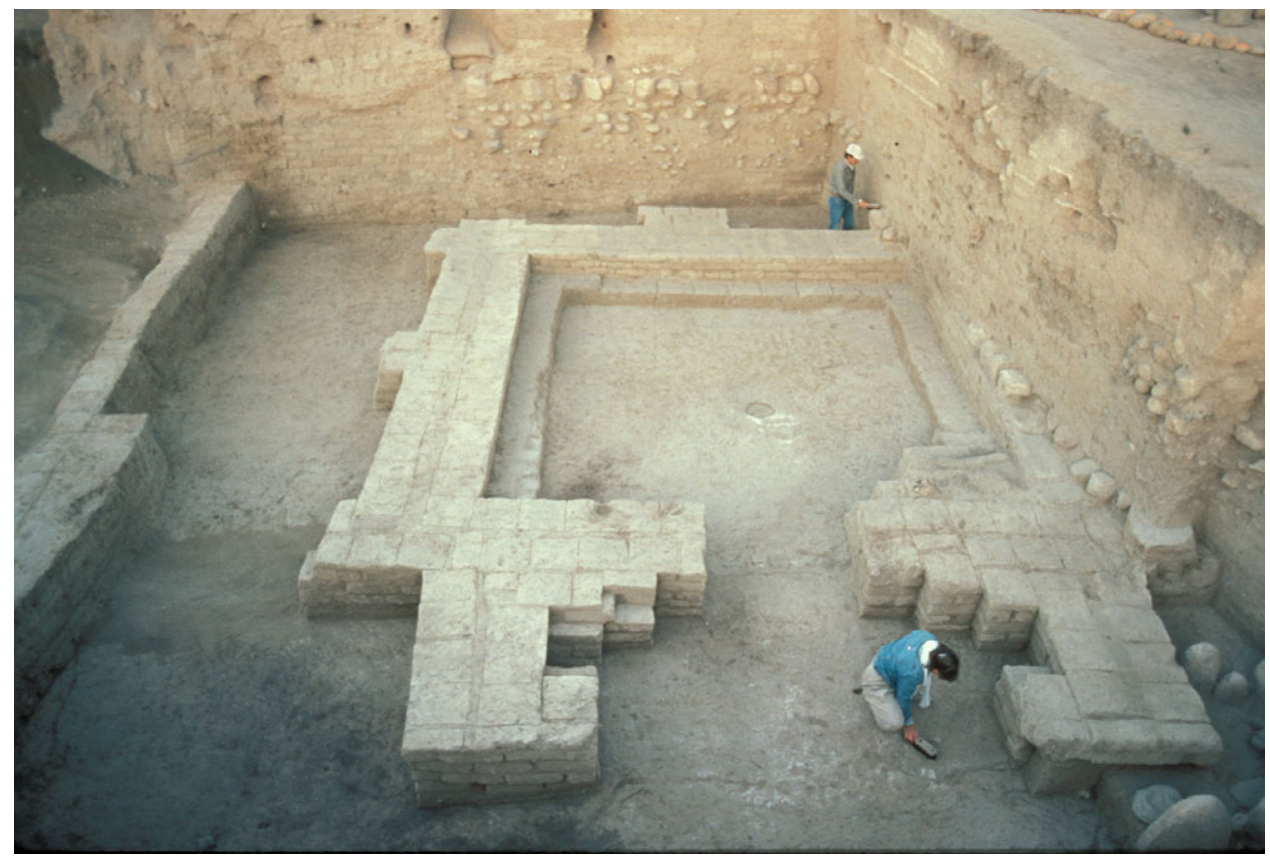

Figure 6 Photo of Phase 4 temple in antis at Tell el-Hayyat, facing west. Temple interior has central depression for pedestal, mudbrick curb, and mudbrick altar in northeast corner. Door framed by buttresses, with standing stones surrounding northern buttress; temple enclosure wall to south.

The architecture and associated community structure at Tell el-Hayyat differs from Tell Abu en-Ni'aj in a number of characteristics. For instance, Tell el-Hayyat's occupants utilized compounds, whether domestic or ritual, that were intentionally segregated from one another by walls and alleys, rather than conjoined in architectural blocks like those of Tell Abu en-Ni'aj. Further, in contrast to the incremental remodeling at Tell Abu en-Ni'aj, the architectural history at Tell el-Hayyat is marked by roughly simultaneous architectural changes across the village between phases. To begin with, Phase 5 is marked clearly by the construction of the earliest temple and its enclosure wall, which lie directly over the nonarchitectural sediments of Phase 6. Subsequently, each of the temples in Phases 5-3 appears to have been intentionally leveled to facilitate its expedient reconstruction. For example, the rammed earth foundations of Phase 5 were shaved flat to provide a level base for the slightly enlarged mudbrick foundations of the Phase 4 temple. The Phase 4 temple walls, in turn, were left standing at a consistent height of five brick courses on top of which the stone foundations for the Phase 3 temple were laid (see Falconer and Fall 2006: figure 3.3). This pattern of intentional leveling continues in Phases 3 and 2, and extends to Tell el-Hayyat's domestic structures as well, such that the entire settlement appears to have been rebuilt en masse in each of the intervals between Phases 5 and 2.

\section{Ceramics and Ceramic Chronologies}

\section{Tell Abu en-Ni'aj}

The most influential interpretive paradigm for Early Bronze IV ceramics stems from Dever's pottery "families" (e.g., 1970a, 1973, 1980), in which site assemblages (primarily from cemeteries) are grouped according to vessel form and decoration both geographically and 
temporally. An associated tripartite chronology of Early Bronze IV A, B and C was predicted to cover 200-300 years at the end of the third millennium BC (Dever 1995) in accordance with a traditional Early Bronze IV time frame. Tell Abu en-Ni'aj provides the prime example of a stratified settlement in the region ascribed to Dever's North Central (NC) family, which he placed in Early Bronze IV B-C (Dever 1980). Among many noteworthy characteristics, Family NC ceramics feature trickle-painted decoration, especially on bowls and jars, which was suggested as a technique introduced from Syria around the middle of Early Bronze IV (Mazzoni 1985).

The ceramic evidence from Tell Abu en-Ni'aj (summarized in Table 3) includes early assemblages (Phases 7-4) characterized by holemouth jars and cook pots, and hemispherical bowls with simple upright rims (Falconer and Fall 2019: 78-86). Many vessel forms (e.g., everted rim jars with smooth shoulder-neck profiles) adhere to Family NC expectations, in contrast to examples to the south (e.g., jars with sharp shoulder-neck profiles) from Jericho (Kenyon and Holland 1983) and Jebel Qa'aqir (Gitin 1975; Dever 2014). However, a number of similarities are apparent between the early phases at Tell Abu en-Ni'aj and more southerly families $\mathbf{J}, \mathrm{CH}$ and $\mathrm{S}$ in holemouth profiles (Palumbo and Peterman 1993), an emphasis on incised, combed and applique decoration (Cohen 1999), and the use of exterior decorative ridges or grooves (Prag 1974, 1986).

The later assemblages from Tell Abu en-Ni'aj (Phases 3-1) conform more closely with the characteristics predicted for Family NC, including the more frequent appearance of open bowls and everted rim jars (Falconer and Fall 2019: 86-101). The frequency of tricklepainting shows its greatest increase between Phases 6 and 5, continues to rise thereafter, and stands in contrast to the predominance of slipped, burnished and rilled decorative techniques seen in more southerly Early Bronze IV families TR and J, for example at Tell Iktanu (Prag 1974), Bab edh-Dhra' (Rast and Schaub 1978, 2003) and Khirbat Iskandar (Richard et al. 2010). Phase 3-1 typological parallels emerge most clearly from northerly mortuary assemblages at Tiwal esh-Sharqi (Helms 1983), el-Hammeh (Wightman 1988), elHusn (Harding and Isserlin 1953), Megiddo (Guy 1938), Beth She'an (Oren 1973) and Tiberias (Tsaferis 1968), as well as the settlement at Tell Um Hammad (Helms 1986; Kennedy 2015). As an additional chronological note, folded envelope ledge handles, the most frequent handle type through all seven phases at Tell Abu en-Ni'aj, do not develop into the vestigial forms expected later in Early Bronze IV (e.g., in Family S; Dever 1970b, 1980).

While prior assignment of Tell Abu en-Ni'aj to Family NC implied its habitation primarily in Early Bronze IV B-C, the mixture of traits associated with more southerly families in Phases 7-4 suggests its founding earlier in the period (i.e., in Early Bronze IVA), while the lack of some late traits connotes abandonment before the end of Early Bronze IV. Based on traditional pottery chronologies, this evidence would predict occupation within a time frame starting after the beginning of Early Bronze IV (i.e., after ca. 2300/2200 BC) and ending before the conclusion of this period (i.e., before ca. 2000 BC).

\section{Tell el-Hayyat}

Among the ceramic evidence from Tell el-Hayyat (summarized in Table 4), Phase 6 deposits at the base of the tell produced exclusively Early Bronze IV pottery typified by hand-built construction, fine grained tempering, and trickle painting as its primary decorative motif, particularly for cups and bowls (Falconer and Fall 2006: 44-46). This assemblage accords 
Table 3 Ceramic descriptions and periods at Tell Abu en-Ni'aj (Falconer and Fall 2019: 75114).

\begin{tabular}{l} 
Phase Period $\quad$ EBIV pottery family affinities \\
\hline Distinctive ceramic types \\
Phases 3-1 FB IVB \\
Bowls \\
Deep bowls: hemispherical forms with upright or slightly inverted tapered rims, trickle- \\
painting sometimes accompanied by horizontal red bands on rim interiors or exteriors, \\
exterior rims occasionally rilled. Open bowls: larger bowls with more strongly everted \\
rims than in Phases 7-4, occasionally with tapered rims, often with flattened rims, rims \\
with more frequent exterior projections and applique decoration, horizontal red bands on \\
rim interiors or exteriors. Majority (55-62\% in each phase) are deep bowls. \\
Jars \\
Everted rim jars: typically with simple rounded rims atop relatively short flaring necks, \\
diagonal incision often on jar shoulders. Holemouth jars: simple flattened, rounded or tapered \\
rims, exterior trickle-painting accompanied by horizontal and vertical bands, and by red \\
horizontal bands on rim exteriors. Majority (75-80\% in each phase) are everted rim jars. \\
Cookpots \\
Short-necked cookpots: simple everted rounded, tapered or flattened rims, some smaller \\
examples have loop handles. Holemouth cookpots: flattened, rounded or internally \\
beveled rims, some rims with internal or external projections or rope molding. Majority \\
(>80\% in each phase) are short-necked cookpots. \\
Noteworthy characteristics: Painting (mostly trickle-painting) more frequent than combing \\
or incision, particularly seen in bowls (both deep and open) and holemouth jars; applique \\
less common than in Phases 7-4; amphoriskoi (often trickle-painted) in Phases 3-1; \\
handles (largely on jars) most commonly are envelope ledge handles \\
(70-90\% in each phase); loop and lug handles on multiple vessel forms; small numbers of \\
four-spouted lamps; ledge handles do not develop into vestigial forms.
\end{tabular}

Phases 7-4 EB IVA Family NC; some affinities with Families J, CH, S.

Bowls

Deep bowls: hemispherical forms with upright or slightly inverted tapered rims, occasionally with rounded, slightly everted rims. Open bowls: commonly upright forms with rounded or flattened rims, sometimes with internal or external grooves below rim; miniature open pinch pot bowls associated with Phase 6 broadroom temple. Slight majority $(51-55 \%$ in Jars each phase) are deep bowls.

Everted rim jars: typically with simple flattened or rounded rims atop relatively short flaring necks; unusual four-handled, flat-bottomed jug in Phase 7. Holemouth jars: commonly with simple flattened rims, sometimes with internal or external projections, some examples with spouts. Majority (60-70\% in each phase) are everted rim jars.

Cookpots

Short-necked cookpots: simple everted rounded or tapered rims. Holemouth cookpots: flattened or internally beveled rims, sometimes with internal or external projections, rims commonly incised or thumb-impressed. Shift from short-necked majorities ( $70-60 \%$ in each phase) in Phases 7 and 6 to holemouth majorities (65-85\% in each phase) in Phases 5 and 4.

Noteworthy characteristics: Combing and incision more frequent than painting (mostly trickle-painting); combing and incision common on bowls (both deep and open) and holemouth jars (including herringbone incision in Phases 6 and 5); applique more common than in Phases 3-1; amphoriskoi (often trickle-painted) in Phases 5 and 4; envelope ledge handles (largely on jars) are most common handle form (50-80\% in each phase); small numbers of four-spouted lamps; unusual seven-spouted lamp in Phase 7. 
best with Dever's families J, N and NC, which have been assigned to Early Bronze IV B-C (Dever 1980). The pottery from stratigraphically contiguous Phase 5 represents a very early Middle Bronze I assemblage characterized by many vessels whose form, fabric and/or decoration combine elements found in classic Early Bronze IV and Middle Bronze I assemblages. In this sense, these vessels may be considered typologically and chronologically transitional. For example, a globular jar from Tell el-Hayyat Phase 5 (e.g., Falconer and Fall 2006: fig. 4.2: g) has a fabric that accords with most of the site's Middle Bronze Age repertoire, while its thickened rim, exterior rilling, and elaborate incision and painting closely parallel those of a jar type found through the final phases of Early Bronze IV at Tell Umm Hammad al-Gharbi (Kennedy 2015). Likewise, Phase 5 bowls commonly combine classic Middle Bronze Age carinated forms with attributes normally found in Early Bronze IV bowls, including slightly incurved rims, external rilling and hand-built construction (see discussion in Falconer and Fall 2006: 46-49, fig. 4.2:c, h, fig. 4.3). The transitional nature of Phase 5 cookpots is best illustrated by coarse-tempered, bell-shaped vessels with thumb-impressed ledge handles (e.g., Falconer and Fall 2006: fig. 4.2: h), which lie typologically between the ledge handles and holemouth forms of Early Bronze IV and the flat-bottomed, straight-sided cookpots of Middle Bronze I. Thus, in light of its transitional pottery and its stratification immediately below the Middle Bronze I deposits in Phase 4, the occupational date of Tell el-Hayyat Phase 5 is ascribed most prudently to very early Middle Bronze I.

Phase 4 pottery provides a classic Middle Bronze I assemblage across a range of functional types, including bowls, juglets, jars and straight-sided cooking pots (Falconer and Fall 2006: 49-52). Vessel forms are consistent with those found in Middle Bronze I assemblages at a variety of Levantine sites, particularly at Jericho (Kenyon and Holland 1982), Lachish (Singer-Avitz 2004), Nahariya (Ben-Dor 1950, 1951) and Pre-Palace Aphek (Beck 2000). The Phase 3 assemblage continues many Middle Bronze I vessel forms, some of which develop in accordance with early Middle Bronze II examples elsewhere (Falconer and Fall 2006: 52-57). Typological parallels continue from a variety of sites, including Jericho (Kenyon and Holland 1982), Pre-Palace Aphek (Beck 2000) and Lachish (Singer-Avitz 2004), plus additional parallels indicative of Middle Bronze II from Shechem (Cole 1984), Megiddo (Ilan 2000) and locally at Tel Beth She'an (Maeir 2010).

Phase 2 presents a hallmark Middle Bronze II-III ceramic assemblage (Falconer and Fall 2006: 56-58). For example, a cache of votive bowls and lamps associated with the last and largest of the Tell el-Hayyat temples (Falconer and Fall 2006: figures 4.9 and 4.10) finds parallels from Middle Bronze II at Tel Dan (Ilan 1996: fig. 4.77) and Lachish (Singer-Avitz 2004: fig.16.1:13), and from Middle Bronze III at Hazor (Garfinkel 1997: fig. III: 12: 10-11), Shechem (Cole 1984: pl. 9: j) and Tel Beth She'an (Maeir 2010). Likewise, juglets and full-sized bowls correspond to Middle Bronze II-III examples from Jericho (Kenyon and Holland 1982: fig. 109, fig. 110: 1-5), Tel Beth She'an (Maeir 2010), Shechem (Cole 1984: pl. 16, pl. 17: a-d), Tel Dan (Ilan 1996: 224, fig. 4: 78) and Hazor (Garfinkel 1997: fig. III: 13: 5). Further clear evidence of a Middle Bronze II-III date for Phase 2 comes from Chocolate-on-White Ware sherds paralleled in Tombs 23 and 24 at nearby Pella (Smith et al. 1981: fig. 26: 7). Chocolate-on-White Ware in the northern Jordan Valley has been dated traditionally between the late 17th and early 16th centuries B.C. (Smith et al. 1981), while more recent work at Tell Abu Kharaz suggests that it ranges from late Middle Bronze II to Late Bronze IB (Fischer 1999, 2006). The Phase 1 ceramics include additional late Middle Bronze Age forms and wares, including subtle rope molding on straight-sided cook pots, 
Table 4 Ceramic descriptions and periods at Tell el-Hayyat (Falconer and Fall 2006: 44-64).

\begin{tabular}{l} 
Phase Period \\
\hline Phase $1 \quad$ MB III \\
Bowls: platter bowls with rolled rims; deep bowls with elongated ribbed necks; ring and \\
disc bases; Jars: juglets with button bases; Cookpots: globular holemouths predominant; \\
flat-bottomed straight-sided form with subtle applique decoration; Noteworthy \\
characteristics: Tell el-Yehudiyeh Ware and Chocolate-on-White Ware sherds. \\
Phase $2 \quad$ MB II/III \\
Bowls: platter bowls with inverted beveled rims; deep bowls with everted pointed rims in \\
rounded, carinated and chalice forms; deep ring bases; Jars: internally ribbed dipper \\
juglets with button or flat bases; Cookpots: globular holemouths predominant; \\
Noteworthy characteristics: Chocolate-on-White painted bowl sherds and tripod bases. \\
Phase $3 \quad$ MB I/II \\
Bowls: platter bowls often with flange below rounded rims; large open bowls with beveled \\
rims; slightly carinated bowls with elongated necks and low disc or ring bases; Jars: \\
narrow mouth jars with elongated everted rims, sometimes with ridge at rim bottom, and \\
with more elaborate profiled rims; wide mouth jars with upright or everted rounded rims; \\
kraters with flattened, commonly rilled rims; Cookpots: shift to globular holemouths with \\
everted rounded rims predominant; flat-bottomed, straight-sided form with diverse styles \\
of applique decoration; perforation of flat-bottomed, straight-sided form less common; \\
Noteworthy characteristics: greatest stylistic diversity of jar rims and cookpot decoration. \\
Phase $4 \quad$ MB I
\end{tabular}

Bowls: platter bowls with beveled, inverted rims; large deep bowls with inverted rims; small carinated bowls with everted rims, in undecorated, slipped, painted or burnished varieties; flat bowl bases most common; Jars: narrow mouth jars with flared, triangular rims, ridged rims or elongated, folded rims; wide mouth jars with inverted or everted profiled rims; globular kraters with thick, rounded or flattened rims; jars commonly without handles; Cookpots: flat-bottomed, straight-sided forms with coarse-tempered fabric; Noteworthy characteristics: first appearance of jars with horizontal combing and profiled jar rims.

Phase 5 early MB I

Bowls: deep bowls with slightly incurved, pointed rims similar to EB IV forms; open platter bowls with beveled rims; thick-bodied, slightly carinated bowls; hand-built, everted rim carinated bowls; Jars: globular kraters with exterior ridges or rills; short-necked everted rim forms; everted tapered-rim forms with EB IV parallels and holemouth forms with flattened rims; transitional EB IV/MB I forms with short necks and thickened rims; Cookpots: hand-built, straight-sided forms; wheel-finished globular forms with everted rims and short necks; Noteworthy characteristics: trickle-painted bowls; rilling and ridging on kraters.

\section{Phase 6 late EB IV}

Bowls: slightly incurved, pointed rims; Jars: holemouth forms with flattened rims; large and small necked forms with everted tapering and rounded rims; folded envelope ledge handles; Noteworthy characteristics: all vessels hand-built; trickle-painting on bowls and jars; thumb-impressed molding on holemouth jar rims and shoulders of necked jars. 
which have Middle Bronze II-III parallels at Shechem (Cole 1984: pl. 23: d) and Jericho (Kenyon and Holland 1982: fig. 147: 8), further specimens of Chocolate-on-White ware, and fragments of Tell el-Yehudiyeh Ware, which peaks in Middle Bronze II-III and continues into the Late Bronze Age (Kaplan 1980).

In sum, the ceramic chronology for Tell el-Hayyat begins with a late Early Bronze IV assemblage in Phase 6, followed immediately by a transitional Phase 5 repertoire that is situated typologically very early in Middle Bronze I. Phases 4 and 3 provide classic Middle Bronze I and transitional Middle Bronze I/II assemblages, respectively. The ceramics from Phases 2 and 1 meet the typological expectations for Middle Bronze II-III assemblages, with specific forms and wares that clearly denote Middle Bronze III habitation at Tell elHayyat. Although there is no Late Bronze Age stratum to cap the Middle Bronze Age sequence at Tell el-Hayyat, the Phase 2 and 1 ceramic assemblages include a variety of vessel forms with clear Middle Bronze III parallels in the Southern Levant, as well as two noteworthy ceramic wares (Chocolate-on-White Ware, Tell el-Yehudiyeh Ware) found through Middle Bronze II-III and into the Late Bronze Age at other Levantine sites. Thus, in terms of ceramic typology, the Phase 5-1 assemblages align with the temporal range of Southern Levantine vessel forms and wares from very early Middle Bronze I into Middle Bronze III, although possibly not to the very end of the Middle Bronze III. Thus, based on traditional Levantine ceramic chronology and Egyptian historical correlations, the Tell el-Hayyat ceramic sequence should start at $2000 \mathrm{BC}$ and continue to $1550 \mathrm{BC}$ or later.

\section{AMS Results and Bayesian Models}

\section{Tell Abu en-Ni'aj}

Radiocarbon determinations from Tell Abu en-Ni'aj now include 25 AMS ages distributed through Phases 7-1 (see Table 1). Phase 7 at Tell Abu en-Ni'aj is represented by a single AMS date (AA-94177) that models about 2500 cal BC. Phase 1 now provides four new radiocarbon ages, all of which model in the 23rd century cal BC. Bayesian analysis coupled with OxCal's Outlier Analysis detected three radiocarbon determinations from Tell Abu en-Ni'aj that met the 5\% threshold in the Outlier Index [O:5/5]: AA-90075 in Phase 6, AA-90071 in Phase 5 and AA-90067 in Phase 4. These samples produce ages sufficiently early to indicate that they most likely represent residual material. All other radiocarbon determinations generate outlier indices $<5 \%$ and are thus treated as representative of their respective stratigraphic contexts. In sum, our Bayesian model indicates that occupation at Tell Abu en-Ni'aj most likely began by 2500 cal BC (based on the start boundary for Phase 7), concluded by 2200 cal BC (based on the end boundary for Phase 1), and spanned up to 300 years (Figure 7).

\section{Tell el-Hayyat}

The recent addition of 13 new dates generated by the Micadas AMS at Groningen now raises the number of ${ }^{14} \mathrm{C}$ ages from Tell el-Hayyat to 31, covering Phases 6-1 (see Table 2). Phase 6 provides three ages that model about 1900 cal BC. Phase 1 is represented by a single age (AA-108793) that models in the late 18th or early 17th century cal BC. OxCal's Outlier Analysis detected two outliers with levels $>5 \%$ : AA-1239 in Phase 5 and GrM-11953 in Phase 2. The Outlier Index reaches $100 / 5$ for these samples, showing both to be anomalously recent and therefore intrusive. AA-1237 reaches the 5\% threshold [O:5/5] and therefore might also be intrusive. All other dates have index scores well below 5, usually between 0 and 1 . These results indicate the earliest deposition at Tell el-Hayyat by 1900 cal 


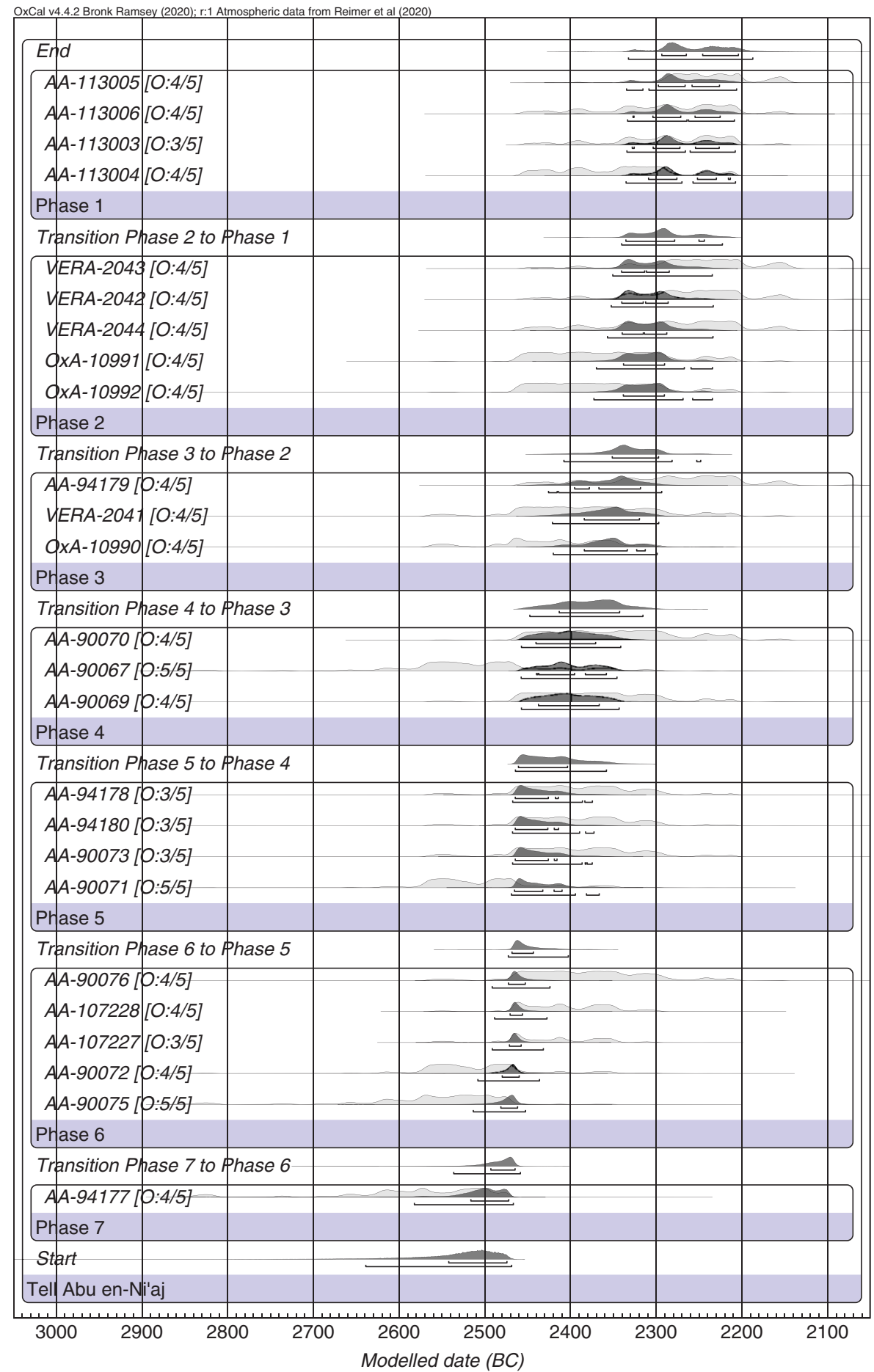

Figure 7 Bayesian sequencing of ${ }^{14} \mathrm{C}$ dates for seed samples from Phases 7-1 at Tell Abu en-Ni'aj, Jordan. Light gray curves indicate single-sample calibration distributions; dark curves indicate modeled calibration distributions. Calibration and Bayesian modeling based on OxCal 4.4.2 (Bronk Ramsey 2009a, 2017) using the IntCal20 atmospheric curve (Reimer et al. 2020). 
BC (based on the Phase 6 start boundary), with early Middle Bronze I occupation starting just after 1900 cal BC (based on the modeled transition between Phases 6 and 5). In conjunction with excavated ceramic evidence, our model suggests a Middle Bronze I/II transition in Phase 3 shortly after $1800 \mathrm{cal} \mathrm{BC}$, and a Middle Bronze II/III transition in Phase 2 between 1800 and $1700 \mathrm{cal}$ BC. These results indicate that Tell el-Hayyat was abandoned by $1600 \mathrm{cal} \mathrm{BC}$ (based on the Phase 1 end boundary), following occupation over approximately 300 years (Figure 8).

\section{DISCUSSION}

\section{Occupational History of Tell Abu en-Ni‘aj and Tell el-Hayyat}

The excavated evidence from Tell Abu en-Ni‘aj portrays a continuously occupied Early Bronze IV agrarian village comprised of mudbrick room blocks built and rebuilt incrementally around a network of earthen and sherd-paved streets. Major iterations of this architectural plan are represented by seven stratified occupational phases and their associated material culture. Based on the affinities of its stratified ceramic assemblages with Dever's pottery families and their chronological assignments, habitation at Tell Abu en-Ni'aj would be predicted to commence after the start of Early Bronze IVA and conclude before the end of Early Bronze IVC.

Bayesian analysis coordinates data from 25 calibrated AMS ages to build a probabilistic model of habitation through seven major phases of roughly comparable lengths (estimated at about 25-40 years each; Table 5), which reaffirms the architectural evidence of fairly steady incremental remodeling within phases and cumulative rebuilding between phases. Our optimal model provides conservative estimates for the founding of Tell Abu en-Ni'aj by about 2500 cal BC and its abandonment by 2200 cal BC. Hence, the exclusively Early Bronze IV ceramic repertoire of Tell Abu en-Ni'aj, pottery-based estimation of habitation beginning in the first portion of this period, and both individual and modeled ${ }^{14} \mathrm{C}$ ages leave little doubt that this village was founded toward the start of Early Bronze IV, no later than 2500 cal BC. Evidence for the latest occupation of Tell Abu en-Ni'aj includes a modeled Phase 1 end boundary prior to 2200 cal BC. Thus, prior pottery-based inference of Tell Abu en-Ni'aj's latest occupation before the end of Early Bronze IV, plus new calibrated radiocarbon determinations, suggest village abandonment by 2200 cal BC.

Tell el-Hayyat contrasts with Tell Abu en-Ni'aj by virtue of its disjunct individual mudbrick structures, its temples as persistent architectural centerpieces, its phase-by-phase rebuilding en masse, and its more variable phase lengths (ranging between about 20 and 70 years each; Table 5). The less consistent phase lengths at Tell el-Hayyat may accord with less incremental, more corporate reconstruction than seen at Tell Abu en-Ni'aj, suggesting the possible influence of central authority (as manifested by the Tell el-Hayyat temples). The relatively modest lengths of Phases 4 and 3 are tempered by a much longer Phase 2, which featured the largest and most elaborate temple. The evidence from Phase 1 includes the fragmentary remains of stone-founded structures on the crown of the tell, but no longer incorporates a village temple.

Based on our Bayesian model, Phase 6 started just before 1900 cal BC and ended shortly after cal 1900 BC. The calibrated dates in immediately subsequent Phase 5 model between 1900 and $1800 \mathrm{cal}$ BC. The distinctly transitional pottery repertoire of Phase 5 marks it as very early Middle Bronze I, which we would expect traditionally to begin about 2000 cal BC. 


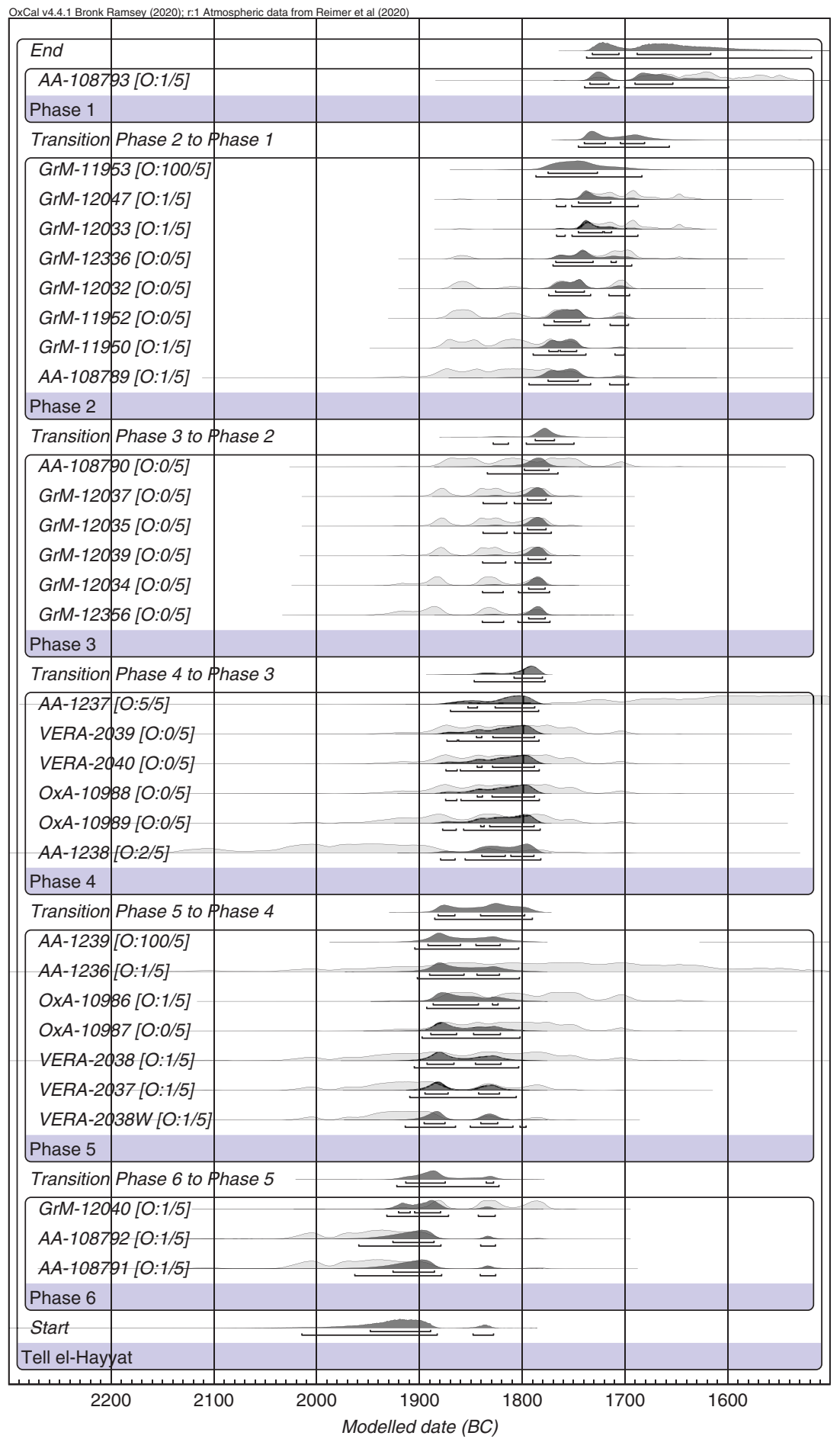

Figure 8 Bayesian sequencing of ${ }^{14} \mathrm{C}$ dates for seed samples from Phases 6-1 at Tell elHayyat, Jordan. Light gray curves indicate single-sample calibration distributions; dark curves indicate modeled calibration distributions. Calibration and Bayesian modeling based on OxCal 4.4.2 (Bronk Ramsey 2009a, 2017) using the IntCal20 atmospheric curve (Reimer et al. 2020). 
Table 5 AMS chronologies and noteworthy features at Tell Abu en-Ni ${ }^{\circ}$ aj and Tell el-Hayyat. Tell Abu en-Ni'aj ceramic ages based on Falconer and Fall 2019: table 1.1. Tell el-Hayyat ceramic ages based on Falconer and Fall 2006: table 4.1. AMS ages (cal yr BC) based on phase boundary medians produced by Bayesian analysis of 31 radiocarbon ages from Tell el-Hayyat and 25 radiocarbon ages from Tell Abu en-Ni'aj (Figures 7 and 8).

Tell el-Hayyat

Phase Period

Ceramic age (BC)

AMS age (cal BC)

Noteworthy features

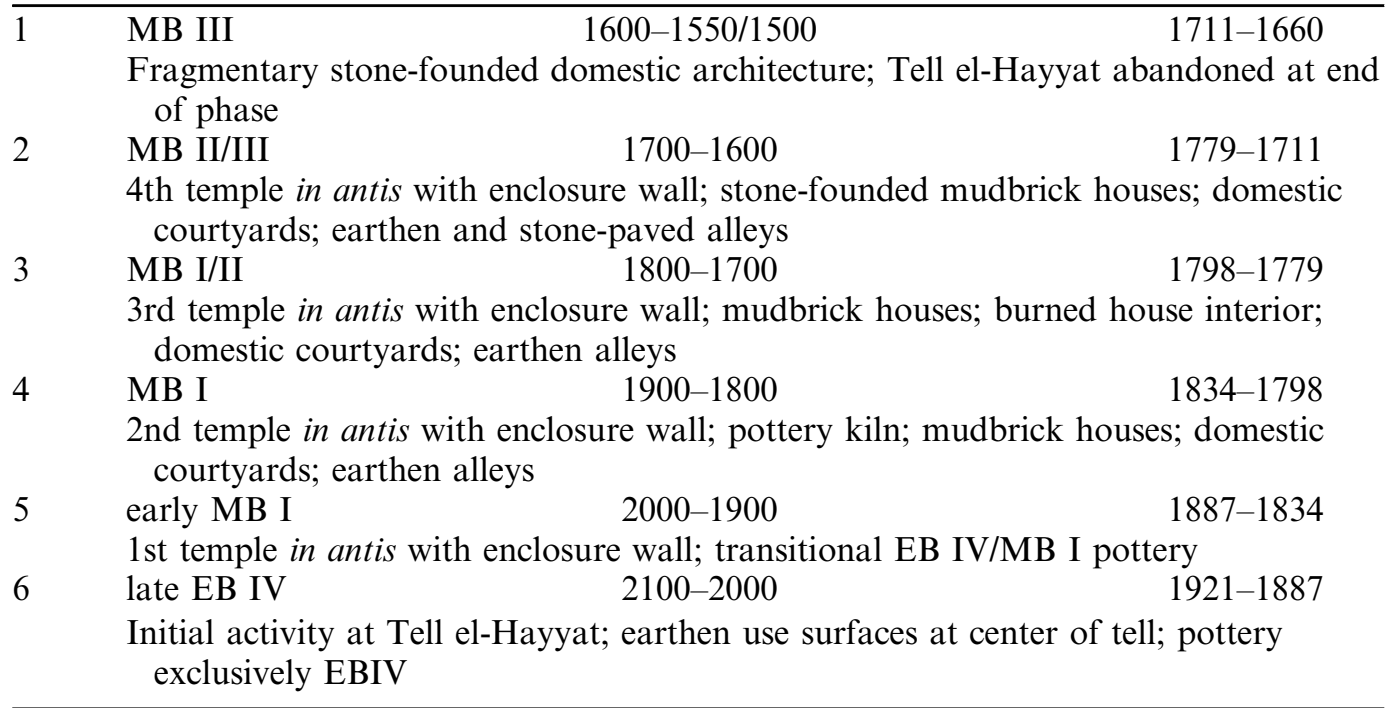

Tell Abu en-Ni'aj

Phase Period

Ceramic age (BC)

AMS age (cal BC)

Noteworthy features

\begin{tabular}{llll}
\hline 1 & EB IVB & $2200-2100$ & 2266
\end{tabular}

Limited mudbrick architecture around sherd-paved intersections in Fields 1 \& 4; Tell Abu en-Ni'aj abandoned at end of phase

2 EB IVB 2200-2100 2331-2293

Agglutinated mudbrick architecture in Fields $1 \& 4$, connected by expanded pattern of broadened sherd-paved streets

3 EB IVB 2200-2100

2373-2331

Agglutinated mudbrick room blocks in Fields 1, 2, 4, linked by repeated \& expanded pattern of sherd-paved streets; large three-basin industrial feature

$4 \quad$ EB IVA $2300-2200$

2415-2373

Agglutinated mudbrick room blocks and individual structures in Field 4, separated by repeated pattern of sherd-paved streets

5 EB IVA 2300-2200

$2452-2415$

Agglutinated mudbrick room blocks in Fields $3 \&$ 4; 1st sherd-paved streets

EB IVA

2300-2200

2483-2452

Broadroom temple with lamb and calf burials in Field 4; individual mudbrick structures

$7 \quad$ EB IVA

2300-2200

$2518-2483$

Initial settlement at Tell Abu en-Ni'aj; sparse mudbrick \& rammed earth structures in Field 4 
In contrast, our model supports a relatively late date for the Early Bronze IV/Middle Bronze I transition at Tell el-Hayyat, quite likely after $1900 \mathrm{cal}$ BC.

Our model places the constituent ages for Phase 4, with its classic Middle Bronze I ceramic assemblage, in the late 19th century cal BC. The dates for Phase 3, with its mix of Middle Bronze I and earlier Middle Bronze II vessel forms, model just after 1800 cal BC. In conjunction with the late Early Bronze/Middle Bronze transition noted above, the combined length for Phases 5, 4 and 3 at Tell el-Hayyat (estimated collectively at somewhat more than a century) represents a later and more compressed time frame than suggested by traditional Levantine chronologies, which tend to allocate about twice this time length to Middle Bronze I alone. Toward the end of Tell el-Hayyat's occupation, Phases 2 and 1 provide ceramic assemblages that include vessel forms and wares characteristic of Middle Bronze III. The seven modeled radiocarbon determinations from Phase 2 (excluding Bayesian outlier GrM-11953) date to the 18th century cal BC, while the lone Phase 1 date and the model end boundary fall in the late 18th or early 17 th century cal BC. Thus, based on current evidence, we conclude that Tell el-Hayyat was abandoned prior to 1600 cal BC.

\section{Regional Implications}

\section{The Early Bronze III/IV Transition}

Modeling of calibrated AMS ages across the Southern Levant proposes comprehensive revision of Early Bronze Age chronology (Regev et al. 2012a) according to which its constituent subperiods are shortened significantly from previous historically-based conventions. From this perspective, the Early Bronze II/III transition would be moved at least two centuries earlier, to around $2900 \mathrm{cal}$ BC, and Early Bronze III would be ended no later than about $2450 \mathrm{cal} \mathrm{BC}$ and as much as a century earlier at some sites. This inference is strengthened by a set of seven AMS seed determinations (presented as three combined ages) from Tell es-Safi (Shai et al. 2014), whereby this site's terminal Early Bronze III deposition, with correspondingly late Early Bronze III pottery forms, is modeled between 2680 and 2580 cal BC (Shai et al. 2014). This inference parallels previous evidence for the end of Early Bronze III from nearby Tel Yarmuth (Regev et al. 2012b) and continues to build the case for a high Early Bronze Age chronology for the Southern Levant. Based on this revised date for the end of Early Bronze III, Regev et al. suggest an overall start date for Early Bronze IV about 2500 cal BC, "even though it could have commenced earlier" (2012a: 561). A mid-third millennium BC beginning for Early Bronze IV is suggested further by radiocarbon ages from settlements at Nahal Refaim, Ein-Ziq, Be'er Resisim and Ha-Gamal in the Southern Levant and Tell Fadous-Kfarabida in Lebanon (Avner and Carmi 2001; Höflmayer et al. 2014; Falconer and Fall 2016: 16-22).

Our new Bayesian model for the occupation of Tell Abu en-Ni'aj clearly accords with a beginning for Early Bronze IV by 2500 cal BC, based a modeled Phase 7 start boundary around or just before 2500 cal BC. Even if we analyze the Tell Abu en-Ni'aj sequence without its single determination from Phase 7, the Phase 6 start boundary is modeled only slightly later, just after 2500 cal BC. The evidence from Tell Abu en-Ni'aj is particularly important in providing a stratified sequence of AMS seed ages that definitively models the founding of this village by about $2500 \mathrm{cal} \mathrm{BC}$ in accordance with a high chronology for the Levantine Early Bronze Age. 


\section{Village Abandonment in Late Early Bronze IV}

Our new Bayesian model for Tell Abu en-Ni'aj also accords with some key expectations of Early Bronze IV ceramic typology and chronology based on Dever's pottery families. Comparison of the Tell Abu en-Ni'aj stratified ceramic assemblages with characteristic vessel forms and decorative motifs found at other sites suggests this village's abandonment before the end of Early Bronze IV. According to our Bayesian model, Phase 1 of Tell Abu en-Ni'aj came to an end by 2200 cal BC (based on the modeled Phase 1 end boundary). A judicious review of Levantine Early Bronze IV AMS ages suggests that the end date for Tell Abu en-Ni'aj may be part of a larger pattern of increasingly pervasive village abandonment across the Levant through the latter portion of this period. For example, southern Levantine Early Bronze IV radiocarbon dates with calibrated medians after 2100 cal BC are limited to four charcoal samples from Bab edh-Dhra' (Beta-134017, median $=2018$ cal BC; SI-2875, median $=1954$ cal BC), Ein-Ziq $($ RT-2514, median = cal 2090 BC) and Nahal Refaim (RT-1711, median = 2040 cal BC) (Rast and Schaub 2003; Avner and Carmi 2001; Segal and Carmi 1996; Regev et al. 2012a; Falconer and Fall 2016: table 4). Early Bronze IV seed ages beyond the Tell Abu en-Ni'aj sequence include seven dates from Tell Mishriefeh, Syria (six from charcoal samples) that run from about 2300 past 2000 cal BC (Bonacossi 2008), three determinations from Tell Arqa in northern Lebanon with calibrated medians between 2302 and 1960 cal BC (Thalmann 2006: 230; 2008), and six ages from Tell Fadous-Kfarabida, in Lebanon south of Tell Arqa, the latest of which has a calibrated median of 2243 cal BC (Genz 2014; Höflmayer et al. 2014). At this point, the best radiocarbon-dated evidence for late Early Bronze IV settlement is limited to the Northern Levant. In contrast, the radiocarbon record for the Southern Levant, and perhaps as far north as Tell Fadous-Kfarabida, features an intriguing dearth of seed-dated evidence later than about $2250 \mathrm{cal} \mathrm{BC}$, and raises the possibility of more common abandonment of southern Levantine villages after that date (see also discussion in D'Andrea and Vacca 2015).

\section{The Early Bronze IV/Middle Bronze Age Interface}

Roughly three centuries of Early Bronze IV habitation at Tell Abu en-Ni'aj were followed by a hiatus prior to the initial settlement of Tell el-Hayyat. Based on their temporally distinctive assemblages and their stratigraphic contiguity, a suite of nine radiocarbon ages from Phases 6 and 5 at Tell el-Hayyat offers an unparalleled opportunity to clarify the beginning of the Middle Bronze Age locally, with potential regional implications. Three Phase 6 AMS dates modeled closely around 1900 cal BC document initial deposition of Early Bronze IV material culture at Tell el-Hayyat. Six Phase 5 ages modeled with similar consistency in the 19th century cal BC capture an immediately subsequent settlement interval very early in Middle Bronze I. The intervening boundary transition lies just after 1900 cal BC. This inference pushes the advent of the Middle Bronze Age in the Jordan Valley appreciably later than the commonly accepted Early Bronze/Middle Bronze chronological benchmark of 2000 BC.

Radiocarbon ages for contexts early in the Middle Bronze Age stem commonly from seed samples, with their accompanying smaller standard deviations and lesser potential for in built age questions (Dee and Bronk Ramsey 2014). The earliest radiocarbon determinations that might support a conventional date about 2000 cal BC for the beginning of the Middle Bronze Age stem from three AMS ages from Gesher (OxA-1955, median= 2014 cal BC), Pella (OZG-611, median $=1995$ cal BC) and Zahrat adh-Dhra' 1 (OZH-756, median = 1992 cal BC) (Garfinkel and Cohen 2007; Bourke et al. 2009; Fall et al. 2019). 
The Pella date is followed by four more ages significantly later in the Middle Bronze Age (Bourke and Zoppi 2007; Bourke et al. 2009), while the age from Gesher reflects a standalone charcoal sample, and the seed date from Zahrat adh-Dhra' 1 is a single disjunct age from a settlement occupied discontinuously primarily later in the Middle Bronze Age (Fall et al. 2019). In contrast, seed-dated AMS sequences through the Middle Bronze Age at the Levantine sites of Jericho, Tel Nami, Tel el-Ifshar and Tell el-Burak all begin after about 1950 cal BC (Falconer and Fall 2016: fig. 8, table 5; Höflmayer et al. 2016b). Our ability to determine a clear date for the beginning of the Middle Bronze Age is hampered by a paucity of sites with contiguous Early Bronze IV/Middle Bronze I stratification such as that found at Tell el-Hayyat. At present, radiocarbon evidence for a conventional date about $2000 \mathrm{cal}$ BC is outweighed by more abundant AMS ages from a greater number of sites in the Southern Levant and Lebanon indicating a more likely beginning date in the range of 1950-1900 cal BC (Höflmayer 2019).

\section{The End of the Middle Bronze Age}

Our updated Bayesian model for Tell el-Hayyat now incorporates 15 AMS ages for Phases 3-1 (excluding one outlier) that document habitation over the latter portion of the Middle Bronze Age. Based on its combination of vessel forms from Middle Bronze I and II, the six tightly clustered Phase 3 dates suggest a transition between these periods shortly after 1800 cal BC. Thus, the evidence from Phases 5-3 caps the length of Middle Bronze I at about one century or perhaps slightly longer. Eight AMS ages through Phases 2 and 1 carry the Tell el-Hayyat chronology into Middle Bronze II-III, with a Phase 2/1 transition in the midst of Middle Bronze III before or just after 1700 cal BC. Village abandonment at the end of Phase 1, later in Middle Bronze III, is modeled clearly before $1600 \mathrm{cal} \mathrm{BC}$.

A Middle Bronze I/II transition about 1800 cal BC is supported by a detailed comparative assessment of architecture, material culture and AMS seed ages from Tel Ifshar and Tell elBurak (Höflmayer et al. 2016b), while a Middle Bronze II/III transition before 1700 cal BC is bolstered by a comparative assessment of multiple lines of evidence from these sites and Tel Kabri on the coast of northern Israel (Höflmayer et al. 2016a). The radiocarbon record from Jericho continues later in the Middle Bronze Age (Bruins and van der Plicht 1995, 2003; Lombardo and Piloto 2000; Nigro et al. 2019), toward a conclusion in the late 17th century cal BC (which would align with Tell el-Hayyat) or in the 16th century cal BC (suggesting asynchronism along the Jordan Rift).

In sum, the chronological inferences stemming from Tell el-Hayyat in conjunction with sites stretching across the Southern Levant and Lebanon provide an empirical basis for beginning the Middle Bronze Age in some locales 50-100 years later than assumed traditionally, an end for some Middle Bronze Age settlements up to a century earlier (Höflmayer 2019), and relocated transitions between the constituent subperiods within this era of rejuvenated town life in the Southern Levant.

\section{CONCLUSIONS}

This study presents and interprets new Bayesian models for Tell Abu en-Ni'aj, based on four new Phase 1 samples and their AMS ages for the final occupation of this Early Bronze IV settlement, and for Tell el-Hayyat, now bolstered by 13 new ${ }^{14} \mathrm{C}$ ages from Early Bronze IV Phase 6 and Phases 3 and 2 in the later Middle Bronze Age. The evidence from Tell Abu en-Ni'aj demonstrates that Early Bronze IV settlement in the Jordan Valley started at 
least as early as $2500 \mathrm{cal} \mathrm{BC}$, and strengthens the argument for a higher Early Bronze IV chronology for the Southern Levant with a start date multiple centuries earlier than assumed traditionally, at least in the Jordan Valley. Our modeling shows that occupation at Tell Abu en-Ni'aj ended by 2200 cal BC, which may hint at a larger pattern of more pervasive village abandonment across the Southern Levant late in Early Bronze IV.

Although the basal stratum at Tell el-Hayyat (Phase 6) is characterized by solely Early Bronze IV sherds, our new modeling shows a substantial gap between the abandonment of Tell Abu en-Ni'aj and the founding of settlement at Tell el-Hayyat. Our modeled start date for very early Middle Bronze Age occupation at Tell el-Hayyat (Phase 5) falls after 1900 cal BC, and represents a significant departure from the standard beginning date for the Middle Bronze Age in the Southern Levant. Bayesian modeling for the later occupation of Tell el-Hayyat, which incorporates newly-augmented suites of AMS ages from Phases 3 and 2, establishes occupation at Tell el-Hayyat by about 1900 cal BC, and suggests the abandonment of this village in Middle Bronze III before 1600 cal BC, which again represents a substantially early departure from standard dates, which presuppose the end of the Middle Bronze Age ca. 1550/1500 cal BC based on conventional synchronization with Egypt.

In tandem, these Bayesian models and their integration with emerging regional radiocarbon chronologies strengthen a set of inferences for Bronze Age settlement in the Jordan Valley and more broadly across the Southern Levant. These inferences indicate (1) further support for a high ${ }^{14} \mathrm{C}$ chronology for Early Bronze IV, (2) abandonment at Tell Abu en-Ni'aj and diminished regional sedentary settlement late in Early Bronze IV, (3) a start date for the Middle Bronze Age more likely at about 1900 cal BC rather than 2000 cal BC, (4) Middle Bronze I/II and II/III transitions around $1800 \mathrm{cal} \mathrm{BC}$ and $1750 \mathrm{cal} \mathrm{BC}$, respectively, and (5) an earlier than expected end date in Middle Bronze III for the abandonment of Tell el-Hayyat by 1600 cal BC. All of these inferences illuminate the need for independent Levantine chronologies and societal interpretations through the Early and Middle Bronze Ages.

\section{ACKNOWLEDGMENTS}

Excavations at Tell Abu en-Ni'aj and Tell el-Hayyat were conducted under permits from the Department of Antiquities, Hashemite Kingdom of Jordan. We offer our thanks to DirectorsGeneral Dr. Adnan Hadidi, Dr. Ghazi Bisheh and Dr. Fawwaz al-Khraysheh. Our fieldwork also benefited from the collaborative support of ACOR Jordan in Amman guided by Directors Dr. David McCreery, Dr. Pierre Bikai and Dr. Barbara Porter. An ACOR Publication Fellowship held by Falconer helped support analysis of Tell Abu en-Ni'aj. Falconer and Fall directed excavations at Tell Abu en-Ni'aj with funding from the National Science Foundation (grants \#SBR 96-00995 and \#SBR 99-04536), the National Geographic Society (\#5629-96) and the Wenner-Gren Foundation for Anthropological Research (\#6006). Excavations at Tell el-Hayyat, directed by Falconer and Bonnie Magness-Gardiner, were funded by grants from the National Endowment for the Humanities (\#RO-21027 and \#RO-22467-92), the National Geographic Society (\#2598-83 and \#2984-84), and the Wenner-Gren Foundation for Anthropological Research (\#4382). Institutional support was provided by the University of Arizona for excavations at Tell el-Hayyat and the first season at Tell Abu en-Ni'aj, and by Arizona State University for the second and third seasons at Tell Abu en-Ni'aj. Radiocarbon dates from Groningen were analyzed in the framework of the Austrian Science Fund (FWF) START-grant Y932-G25 "Tracing Transformations" directed by Felix Höflmayer. This publication of research results from Tell Abu en-Ni‘aj and Tell 
el-Hayyat was supported by a grant to Falconer and Fall from the National Science Foundation (\#1850259). We thank two anonymous reviewers for their helpful comments, Patrick Jones for drafting Figure 1, and Wei Ming for drafting Figures 2, 3, and 5.

\section{REFERENCES}

Avner U, Carmi I. 2001. Settlement patterns in the southern Levant deserts during the 6th-3rd millennia $\mathrm{BC}$ : a revision based on ${ }^{14} \mathrm{C}$ dating. Radiocarbon 43(3):1203-1216.

Beck P. 2000. The Middle Bronze Age IIA pottery repertoire: a comparative study. In: Kochavi M, Beck P, Yadin E, editors. Aphek-Antipatris I: Excavation of Areas A and B - The 1972-1976 Seasons. Tel Aviv: Tel Aviv University, Sonia and Marco Nadler Institute of Archaeology. p. 239-254.

Ben-Dor I. 1950. A Middle-Bronze Age temple at Nahariya. Quarterly of the Department of Antiquities in Palestine 24:1-42.

Ben-Dor I. 1951. A Canaanite temple at Nahariyya. Eretz-Israel 1:17-28

Bietak M. 2013. Antagonisms in historical and radiocarbon chronology. In: Shortland AJ, Bronk Ramsey C, editors. Radiocarbon and the chronologies of Ancient Egypt 16. London: Oxbow. p. 76-109.

Bonacossi DM. 2008. The EB/MB transition at Tell Mishrifeh: stratigraphy, ceramics and absolute chronology. A preliminary review. In: Bietak M, Czerny E, editors. The Bronze Age in the Lebanon. Studies on the Archaeology and Chronology of Lebanon, Syria and Egypt. Vienna: Verlag der Osterreichischen Akademie der Wissenschaften. p. 127-152.

Bourke SJ. 2014. The southern Levant (Transjordan) during the Middle Bronze Age. In: Steiner ML, Killebrew AE, editors. The Archaeology of the Levant c. 8000-332 BCE. Oxford: Oxford University Press. p. 463-481.

Bourke SJ, Zoppi U. 2007. Dating the Cultic Assemblages from the Bronze Age Fortress Temple Complex at Pella in Jordan. Progress Report for AINGRA 05013. Sydney: University of Sydney.

Bourke SJ, Zoppi U, Hua Q, Meadows J, Gibbins S. 2009. The beginning of the Early Bronze Age in the north Jordan Valley: new ${ }^{14} \mathrm{C}$ determinations from Pella in Jordan. Radiocarbon 51(3):905-913.

Bronk Ramsey C. 2009a. Bayesian analysis of radiocarbon dates. Radiocarbon 51(1):337-360.

Bronk Ramsey C. 2009b. Dealing with outliers and offsets in radiocarbon dating. Radiocarbon 51(3):1023-1045.

Bronk Ramsey C. 2017. Methods for summarizing radiocarbon datasets. Radiocarbon 59(2): 1809-1833.

Bronk Ramsey C, Dee MW, Rowland JM, Higham TFG, Harris SA, Brock F, Quiles A, Wild EM,
Marcus ES, Shortland AJ. 2010. Radiocarbonbased chronology for dynastic Egypt. Science 328(5985):1554-1557.

Bruins H. 2007. Charcoal radiocarbon dates of Tell el-Dab'a. In: Bietak M, Czerny E, editors. The Synchronisation of Civilisations in the Easter Mediterranean in the Second Millennium B.C. III, Proceedings of the SCIEM 2000 2nd EuroConference Vienna, 28th May-1st June 2003. Wien: Verlag der Osterreichischen Akademie der Wissenschaften. p. 65-77.

Bruins H, van der Plicht J. 1995. Tell es-Sultan (Jericho): Radiocarbon results of the short-lived cereal and multiyear charcoal samples from the end of the Middle Bronze Age. Radiocarbon 37:213-220.

Bruins H, van der Plicht J. 2001. Radiocarbon challenges archaeo-historical time frameworks in the Near East: the Early Bronze Age of Jericho in relation to Egypt. Radiocarbon 43(3):1321-1332.

Bruins H, van der Plicht J. 2003. Assorting and Synchronising Archaeological and Geological Strata with Radiocarbon: The Southern Levant in relation to Egypt and Thera. In: Bietak M, editor. The synchronisation of civilisations in the Eastern Mediterranean in the second Millennium B.C. II. Proceedings of the SCIEM 2000-EuroConference Haindorf, 2nd of May-7th of May 2001, Contributions to the Chronology of the Eastern Mediterranean 4. Vienna: Verlag der Osterreichischen Akademie der Wissenschaften. p. 35-42.

Burke AA. 2008. "Walled Up to Heaven". The evolution of Middle Bronze Age fortification strategies in the Levant. Studies in the Archaeology and History of the Levant 4 . Winona Lake: Eisenbrauns.

Burke AA. 2014. Introduction to the Levant during the Middle Bronze Age. In: Steiner ML, Killebrew AE, editors. The archaeology of the Levant c. 8000-332 BCE. Oxford: Oxford University Press. p. 403-413.

Cohen R. 1999. Ancient Settlement of the Central Negev. Volume 1. The Chalcolithic Period, The Early Bronze Age I. IAA Reports 6. Jerusalem: Israel Antiquities Authority. In Hebrew.

Cohen SL. 2002. Canaanites, chronologies, and connections, the relationship of Middle Bronze IIA Canaan to Middle Kingdom Egypt. Studies in the Archaeology and History of the Levant 3. Winona Lake: Eisenbrauns.

Cohen SL. 2009. Continuities and discontinuities: a re-examination of the Intermediate Bronze AgeMiddle Bronze Age transition in Canaan. 
Bulletin of the American Schools of Oriental Research 354:1-13.

Cohen SL. 2014. The southern Levant (Cisjordan) during the Middle Bronze Age. In: Steiner ML, Killebrew AE, editors. The Archaeology of the Levant c. 8000-332 BCE. Oxford: Oxford University Press. p. 451-464.

Cohen SL. 2016. Peripheral Concerns. Urban development in the Bronze Age Southern Levant. Sheffield, Bristol: Equinox.

Cole DP. 1984. Shechem I: The Middle Bronze IIB Pottery. Winona Lake, IN: American Schools of Oriental Research.

D'Andrea M. 2014. The Southern Levant in Early Bronze IV. Issues and perspectives in the pottery evidence. Contributi e Materiali di Archeologia Orientale XVII, Roma: Sapienza Università di Roma.

D'Andrea M, Vacca A. 2015. The northern and southern Levant during the late Early Bronze Age: a reappraisal of the "Syrian Connection." In: Matthiae P, editor. Studia Eblaitica 1. Studies on the Archaeology, History, and Philology of Ancient Syria. Wiesbaden: Harrassowitz Verlag. p. 43-73.

Dee MW, Bronk Ramsey C. 2014. High-precision Bayesian modeling of samples susceptible to inbuilt age. Radiocarbon 56(1):83-94.

Delorit RJ. 1970. Illustrated taxonomy manual of weed seeds. River Falls, WI: Agronomy Publications.

de Miroschedji P. 2009. Rise and collapse in the southern Levant in the Early Bronze Age. In: Cardarelli A, Cazzella A, Frangipane M, Peroni $\mathrm{R}$, editors. Reasons for change: birth, decline and collapse of societies from the end of the fourth to the beginning of the first millennium BC. Universita degli Studi di Roma 'La Sapienza'. Rome. p. 101-129.

de Miroschedji P. 2014. The southern Levant (Cisjordan) during the Early Bronze Age. In: Steiner ML, Killebrew AE, editors. The Archaeology of the Levant c. 8000-332 BCE. Oxford: Oxford University Press. p. 307-329.

Dever WG. 1970a. The 'Middle Bronze I' Period in Syria and Palestine. In: Sanders JA, editor. Near Eastern archaeology in the twentieth century; essays in honor of Nelson Glueck. New York: Double Day and Company Inc. p. 132-163.

Dever WG. 1970b. Vestigial features in MB I: an illustration of some principles of ceramic typology. Bulletin of the American Schools of Oriental Research 200:19-30.

Dever WG. 1973. The EB IV-MBI Horizon in Transjordan and Southern Palestine. Bulletin of the American Schools of Oriental Research 210:37-63.

Dever WG. 1980. New Vistas on the EBIV (MBI) Horizon in Syria-Palestine. Bulletin of the American Schools of Oriental Research 237:35-64.
Dever WG. 1987. The Middle Bronze Age: the zenith of the urban Canaanite era. Biblical Archaeologist 50:149-177.

Dever WG. 1995. Social structure in the Early Bronze IV period in Palestine. In: Levy TE, editor. The Archaeology of Society in the Holy Land. New York: Facts on File. p. 282-296.

Dever WG. 2014. Excavations at the Early Bronze IV Sites of Jebel Qa'aqir and Be'er Resisim. Studies in the Archaeology and History of the Levant 6. Winona Lake: Eisenbrauns.

Falconer SE, Fall PL. 2006. Bronze Age rural ecology and village life at Tell el-Hayyat, Jordan. British Archaeological Reports, International Series 1586. Oxford: Archaeopress.

Falconer SE, Fall PL. 2009. Settling the valley: agrarian settlement and interaction along the Jordan Rift during the Bronze Age. In: Kaptijn E, Petit L, editors. A timeless vale: archaeological and related essays on the Jordan Valley. Archaeological Studies, Leiden University 19. Leiden: Leiden University Press. p. 97-107.

Falconer SE, Fall PL. 2016. A radiocarbon sequence from Tell Abu en-Ni'aj, Jordan and its implications for Early Bronze IV chronology in the Southern Levant. Radiocarbon 58(3):615647. doi: 10.1017/RDC.2016.26.

Falconer SE, Fall PL. 2017. Radiocarbon evidence from Tell Abu en-Ni'aj and Tell el-Hayyat, Jordan and its implications for Bronze Age Levantine and Egyptian chronologies. Journal of Ancient Egyptian Interconnections 13:7-19.

Falconer SE, Fall PL. 2019. Early Bronze IV village life in the Jordan Valley: excavations at Tell Abu en-Ni'aj and Dharet Umm el-Marar, Jordan. British Archaeological Reports, International Series, 2922, Oxford: BAR Publishing.

Fall PL, Lines L, Falconer SE. 1998. Seeds of civilization: Bronze Age rural economy and ecology in the Southern Levant. Annual of the Association of American Geographers 88:107-125.

Fall PL, Falconer SE, Lines L. 2002. Agricultural intensification and the secondary products revolution along the Jordan Rift. Human Ecology 30:445-482.

Fall PL, Falconer SE, Klinge J. 2015. Bronze Age fuel use and its implications for agrarian landscapes in the eastern Mediterranean. Journal of Archaeological Science Reports 4:182-191. doi: 10.1016/j.jasrep.2015.09.004.

Fall PL, Falconer SE, Porson S. 2019. Archaeobotanical Inference of Intermittent Settlement and Agriculture at Middle Bronze Age Zahrat adh-Dhra' 1, Jordan. Journal of Archaeological Science: Reports 26. doi: 10. 1016/j.jasrep.2019.101884.

Fischer PM 1999. Chocolate-on-White ware: typology, chronology and provenance-the evidence from Tell Abu al-Kharaz, Jordan Valley. Bulletin of the American Schools of Oriental Research 313:1-29. 
Fischer PM. 2006. The rise and fall of Middle and Late Bronze Age societies of Tell Abu alKharaz. In: Fischer PM, editor. The chronology of the Jordan Valley during the Middle and Late Bronze Ages: Pella, Tell Abu al-Kharaz and Tell Deir Alla. Vienna: Contributions to the Chronology of the Eastern Mediterranean 12. p. $59-197$.

Fowler DM, Fayek M, Middleton E. 2011. Clay acquisition and processing strategies during the first millennium A.D. in the Thukela River Basin, South Africa: an ethnoarchaeological approach. Journal of Archaeological Science 26(5):762-785.

Garfinkel Y. 1997. The Middle and Late Bronze Age phases in Area L. In: Ben-Tor A, Bonfil R, editors. Hazor V: an account of the fifth season of excavations, 1968. Jerusalem: The Israel Exploration Society, The Hebrew University of Jerusalem. p. 194-217.

Garfinkel Y, Cohen S, editors. 2007. The Middle Bronze IIA cemetery at Gesher: final report. Boston: Annual of the American Schools of Oriental Research 62.

Genz H. 2014. Excavations at Tell Fadous-Kfarabida 2004-2011: an Early and Middle Bronze Age site on the Lebanese coast. In: Höflmayer F, Eichmann R, editors. Egypt and the Southern Levant in the Early Bronze Age. OrientArchäologie 31. Rahden: Verlag Marie Leidorf. p. 69-91.

Gitin S. 1975. Middle Bronze I 'Domestic Pottery' at Jebel Qa'aqir: a ceramic inventory of Cave G23. Eretz Israel 12:46-62.

Glueck N. 1951. Explorations in eastern Palestine IV. Annual of the American Schools of Oriental Research 25-28. New Haven: American Schools of Oriental Research.

Gophna R. 1995. Early Bronze Age Canaan: some spatial and demographic observations. In: Levy TE, editor. The Archaeology of Society in the Holy Land. New York: Facts on File. p. 269-281.

Golani A, Segal D. 2002. Redefining the onset of the Early Bronze Age in Southern Canaan: new ${ }^{14} \mathrm{C}$ evidence from Ashqelon Afridar. In: van den Brink E, Yannai E, editors. In quest of ancient settlements and landscapes. Tel Aviv: Ramot Publishing. p. 135-154.

Greenberg R. 2002. Early urbanizations in the Levant: a regional narrative. London: Leicester University Press.

Greenberg R. 2014. Introduction to the Levant during the Early Bronze Age. In: Steiner ML, Killebrew AE, editors. The Archaeology of the Levant c. 8000-332 BCE. Oxford: Oxford University Press. p. 269-277.

Guy PLO. 1938. Megiddo tombs. Chicago: University of Chicago Press. Oriental Institute Publications 33.
Harding GL, Isserlin, BSJ. 1953. An Early Bronze Age cave at el-Husn. Palestine Exploration Fund Quarterly 6:1-13.

Helbaek H. 1958. Appendix A. Plant economy in ancient Lachish. In: Tufnell O, editor. Lachish: Tell Ed Duweir 4, the Bronze Age. Oxford: Oxford University Press. p. 309-317.

Helbaek H. 1966. The plant remains from Nimrud. In: Mallowan MEL, editor. Nimrud and its Remains, Appendix I, Vol. 2. London: Collins. p. 613-620.

Helms SW. 1983. The EB IV (EB-MB) Cemetery at Tiwal esh-Sharqi, in the Jordan Valley. Annual of the Department of Antiquities of Jordan 27: 55-85.

Helms SW. 1986. Excavations at Tell Um Hammad, 1984. Levant 18:25-50.

Höflmayer F. 2019. The expulsion of the Hyksos and the end of the Middle Bronze Age: a reassessment in light of recent chronological research. Journal of Ancient Egyptian Interconnections 21:20-30.

Höflmayer F, Dee MW, Genz H, Riehl S. 2014. Radiocarbon evidence for the Early Bronze Age Levant: the site of Tell Fadous-Kfarabida (Lebanon) and the end of the Early Bronze III Period. Radiocarbon 56(2):529-542.

Höflmayer F, Yasur-Landau A, Cline EH, Dee MW, Lorentzen B, Riehl S. 2016a. New Radiocarbon dates from Tel Kabri support a high Middle Bronze Age chronology. Radiocarbon 58(3):599-613.

Höflmayer F, Kamlah J, Sader H, Dee MW, Kutschera W, Wild EM, Riehl S. 2016b. New Evidence for Middle Bronze Age chronology and synchronisms in the Levant: radiocarbon dates from Tell el-Burak, Tell el-Dab'a, and Tel Ifshar Compared American Schools of Oriental Research. Bulletin of the American Schools of Oriental Research 375:53-76.

Hubbard RNLB. 1992. Dichotomous keys for the identification of the major Old World crops. Review of Palaeobotany and Palynology 73:109-115.

Ibrahim M, Sauer J, Yassine K. 1976. The East Jordan Valley Survey, 1975. Bulletin of the American Schools of Oriental Research 222:41-66.

Ilan D. 1995. "The Dawn of Internationalism-The Middle Bronze Age." In: Levy TE, editor. The archaeology of society in the Holy Land. New York: Facts on File. p. 297-319.

Ilan D. 1996. The Middle Bronze Age tombs. In: Biran A, editor. Dan I. Jerusalem: Nelson Glueck School of Biblical Archaeology, Hebrew Union College - Jewish Institute of Religion.

Ilan D. 2000. The Middle Bronze Age pottery. In: Finkelstein I, Ussishkin D, Halpern B editors. Megiddo III, the 1992-1996 seasons. Tel Aviv: Emery and Claire Yass Publications in Archaeology, the Institute of Archaeology, Tel Aviv University. p. 186-222. 
Jacomet S. 2006. Identification of cereal remains from archaeological sites, 2nd edition. Archaeobotany Lab, IPAS. Basel: Basel University.

Joffe A. 1993. Settlement and Society in the Early Bronze Age I and II, Southern Levant. Sheffield: Sheffield Academic Press.

Kaplan MF. 1980. The Origin and Distribution of Tell el-Yehudiyeh Ware, Studies in Mediterranean Archaeology 42. Göteborg: Paul Åström.

Kapellakis I, Tsagarakis KP, Crowther JC. 2008. Olive oil history, production and by-product management. Reviews in Environmental and Bio/Technology 7(1):1-26.

Kennedy MA. 2015. Assessing the Early BronzeMiddle Bronze Age transition in the Southern Levant in light of a transitional ceramic vessel from Tell Umm Hammad, Jordan. Bulletin of the American Schools of Oriental Research 373:199-216.

Kenyon KM, Holland TA. 1982. Excavations at Jericho 4, London: British School of Archaeology in Jerusalem.

Kenyon KM, Holland TA. 1983. Excavations at Jericho 5, London: British School of Archaeology in Jerusalem.

Klinge J, Fall PL. 2010. Archaeobotanical inference of Bronze Age land use and land cover in the eastern Mediterranean. Journal of Archaeological Science 37:2622-2629.

Kramer C. 1982. Village ethnoarchaeology. Rural Iran in archaeological perspective. New York: Academic Press.

Kutschera W, Bietak M, Wild EM, Bronk Ramsey C, Dee M, Golser R, Kopetsky K, Stadler P, Steier P, Thanheiser U, Weninger F. 2012. The chronology of Tell el-Daba: a crucial meeting point of ${ }^{14} \mathrm{C}$ dating, archaeology, and Egyptology in the 2nd millennium BC. Radiocarbon 54(3-4):407-422.

Lombardo M, Piloto A. 2000. Appendix D: new radiocarbon dates and assessment of all dates obtained for the Early and Middle Bronze ages in Jericho. In: Marchetti N, Nigro L, editors. Excavations at Jericho, 1998. Preliminary report on the second season of excavations and surveys at Tell es-Sultan. Quaderni di Gerico 2. Roma: Università di Roma "La Sapienza.” p. 329-332.

Maeir AM. 2010. In the midst of the Jordan. The Jordan Valley during the Middle Bronze Age (circa 2000-1500 BCE). Archaeological and historical correlates. Contributions to the chronology of the Eastern Mediterranean 26. Wien: Verlag der Österreichischen Akademie der Wissenschaften.

Magness-Gardiner B, Falconer SE. 1994. Community, polity, and ritual in a Middle Bronze Age Levantine village. Journal of Mediterranean Archaeology 7(2):3-40.

Manning SW, Höflmayer F, Moeller N, Dee MW, Bronk Ramsey C, Fleitmann D, Higham T, Kutschera W, Wild E.M. 2014. Dating the
Thera (Santorini) eruption: archaeological and scientific evidence supporting a high chronology. Antiquity 88:1164-1179.

Martin AC, Barkley WD. 1973. Seed identification manual. 2nd ed. Berkeley: University of California Press.

Mazzoni S. 1985. Frontieres ceramiques et le Haut Euphrate au Bronze Ancien IV. Mari Annales de Recherches Interdisciplinaire 4:561-577.

Mellart J. 1962. Preliminary report on the archaeological survey in the Yarmuk and Jordan Valley for the Point Four irrigation scheme. Annual of the Department of Antiquities of Jordan 6-7:126-157.

Nigro L, Calcagnile L, Yasin J, Gallo E, Quarta G. 2019. Jericho and the chronology of Palestine in the Early Bronze Age: A radiometric re-assessment. Radiocarbon 61(1):211-241.

Oren E. 1973. The Northern Cemetery of Beth Shean. Leiden: Brills.

Palumbo G. 1991. The Early Bronze Age IV in the Southern Levant: Settlement Patterns, Economy and Material Culture of a "Dark Age". Contributi Materiali di Archeologia Orientale III (1990). Rome: Universita Degli Studi di Roma la Sapienza.

Palumbo G, Peterman G. 1993. Early Bronze Age IV ceramic regionalism in central Jordan. Bulletin of the American Schools of Oriental Research 289:23-32.

Philip G. 2003. The Early Bronze Age of the Southern Levant: a landscape approach. Journal of Mediterranean Archaeology 16(1):103-132.

Philip G. 2008. The Early Bronze I-III Age. In: Adams R, editor. The Archaeology of Jordan: a reader. London: Equinox. p. 161-226.

Porson S, Fall PL, Falconer SE. 2019. Archaeobotanical analyses of carbonized plant remains from Tell $\mathrm{Abu}$ en-Ni'aj. In: Falconer SE, Fall PL. Early Bronze IV village life in the Jordan Valley: excavations at Tell Abu en-Ni'aj and Dharet Umm el-Marar, Jordan. British Archaeological Reports, International Series, 2922, Oxford: BAR Publishing. p. 131-138.

Prag K. 1974. The Intermediate Early Bronze-Middle Bronze Age: an interpretation of the evidence from Transjordan, Syria, and Lebanon. Levant 6:69-116.

Prag K. 1986. The Intermediate Early Bronze-Middle Bronze Age sequences at Jericho and Tell Iktanu reviewed. Bulletin of the American Schools of Oriental Research 264:61-72.

Prag K. 2001. The third millennium in Jordan: A perspective, past and future. In: al-Khraysheh F, editor. Studies in the History and Archaeology of Jordan. Vol. 7. Amman, Jordan: Department of Antiquities. p. 179-190.

Prag K. 2014. The southern Levant during the Intermediate Bronze Age. In: Steiner ML, Killebrew AE, editors. The archaeology of the 
Levant c. 8000-332 BCE. Oxford: Oxford University Press. p. 388-400.

Rast WE, Schaub RT. 1978. A preliminary report of excavations at Bab edh-Dhra', 1975. Annual of the American Schools of Oriental Research 43:1-32.

Rast WE, Schaub RT. 2003. Bâb edh-Dhrâ: Excavations at the Town Site (1975-1981). Eisenbrauns: Winona Lake.

Regev J, de Miroschedji P, Greenberg R, Braun E, Greenhut Z, Boaretto E. 2012a. Chronology of the Early Bronze Age in the Southern Levant: new analysis for a high chronology. Radiocarbon 54(3-4):525-566.

Regev J, de Miroschedji P, Boaretto E. 2012b. Early Bronze Age chronology: radiocarbon dates and chronological models from Tel Yarmuth (Israel). Radiocarbon 54(3-4):505-524.

Regev J, Finkelstein I, Adams MJ, Boaretto E. 2014. Wiggle-matched ${ }^{14} \mathrm{C}$ chronology of Early Bronze Megiddo and the synchronization of Egyptian and Levantine chronologies. Agypten und Levante 24:243-266.

Reimer PJ, Austin, WEN, Bard E, Bayliss A, Blackwell PG, Bronk Ramsey C, Butzin M, Cheng H, Edwards RL, Friedrich M, Grootes PM, Guilderson TP, Hajdas I, Heaton TJ, Hogg AG, Hughen KA, Kromer B, Manning SW, Muscheler R, Palmer JG, Pearson C, van der Plicht J, Reimer RW, Richards DA, Scott EM, Southon JR, Turney CSM, Wacker L, Adolphi F, Buntgen U, Capano M, Fahrni SM, Fogtmann-Schulz A, Friedrich R, Köhler P, Kudsk S, Miyake F, Olsen J, Reinig, Sakamoto M, Sookdeo A, Talamo S. 2020. The IntCal20 northern hemisphere radiocarbon age calibration curve (0-55 kBP). Radiocarbon 62 . doi: 10.1017/RDC.2020.41.

Richard S. 2014. The southern Levant (Transjordan) during the Early Bronze Age. In: Steiner ML, Killebrew AE, editors. The archaeology of the Levant c. 8000-332 BCE. Oxford: Oxford University Press. p. 330-352.

Richard S, Long JC Jr, Holdorf PS, Peterman G. 2010. Khirbat Iskandar. Final report on the Early Bronze IV Area C "Gateway" and Cemeteries. American Schools of Oriental Research Archaeological Reports 14. Boston: American Schools of Oriental Research.

Segal D, Carmi I. 1996. Rehovot radiocarbon date list V. 'Atiqot XXIX:79-106.

Shai I, Greenfield HJ, Regev J, Boaretto E, EliyahuBehar A, Maeir AM. 2014. The Early Bronze Age remains at Tell es-Safi/Gath: an interim report. Tel Aviv 41(1):20-49.

Sharon 2014. Levantine chronology. In: Steiner ML, Killebrew AE, editors. The Archaeology of the
Levant c. 8000-332 BCE. Oxford: Oxford University Press. p. 44-65.

Singer-Avitz L. 2004. The Middle Bronze Age pottery from Areas D and P. In Ussishkin D, editor. The renewed archaeological excavations at Lachish (1973-1994). Vol. 3. Tel Aviv: Emery and Claire Yass Publications in Archaeology, Tel Aviv University. p. 900-970.

Smith RH, McNicoll AW, Hennessy JB. 1981. The 1980 season at Pella of the Decapolis. Bulletin of the American Schools of Oriental Research 245:1-30.

Sowada KN. 2009. Egypt in the Eastern Mediterranean during the Old Kingdom. An archaeological perspective. Orbis Biblicus et Orientalis 237. Fribourg, Göttingen: Academic Press; Vandenhoeck \& Ruprecht.

Stager LE. 1992. The periodization of Palestine from Neolithic through Early Bronze Age times. In: Ehrich RW, editor. Chronologies in Old World Archaeology. 3rd edition. Chicago: University of Chicago Press. Volume 1:22-41; Volume 2:17-60.

Thalmann J-P. 2006. Tell Arqa - I. Les niveaux de l'age du Bronze. Vol. 1. Beirut: Institut Francais du Proche-Orient.

Thalmann J-P. 2008. Tell Arqa et Byblos, essai de correlation. In: Bietak M, Czerny E, editors. The Bronze Age in the Lebanon. Studies on the Archaeology and Chronology of Lebanon, Syria and Egypt. Vienna: Verlag der Osterreichischen Akademie der Wissenschaften. p. 61-78.

Tsaferis V. 1968. A Middle Bronze Age I cemetery in Tiberias. Israel Exploration Journal 18(1): 15-19.

van der Plicht J, Bronk Ramsey C, Heaton TJ, Scott EM, Talamo S. 2020. Recent developments in calibration for archaeological and environmental samples. Radiocarbon 62. doi: 10.1017/RDC.2020.22.

van Zeist W. 1976. On macroscopic traces of food plants in southwestern Asia (with some reference to pollen data). Philosophical Transactions of the Royal Society London B 275:27-41.

van Zeist W, Bakker-Heeres JAH. 1982. Archaeobotanical studies in the Levant. 1. Neolithic sites in the Damascus basin: Aswad, Ghoraife, Ramad. Palaeohistoria 24:165-256.

Waterbolk HT. 1971. Working with radiocarbon dates. Proceedings of the Prehistoric Society 37(2):15-31.

Wightman GJ. 1988. An EB IV Cemetery in the North Jordan Valley. Levant 20:139-59.

Zohary D, Hopf M. 1973. Domestication of pulses in the Old World. Science 182:887-894.

Zohary D, Spiegel-Roy P. 1975. Beginnings of fruit growing in the Old World. Science 187:319-327. 